\title{
Global stability and bounds for coarsening rates within the mean-field theory for domain coarsening
}

\author{
B. Niethammer ${ }^{1}$ and J. J. L. Velázquez ${ }^{2}$
}

\begin{abstract}
We investigate the long-time behavior of solutions to the classical mean-field model for coarsening by Lifshitz-Slyozov and Wagner (LSW). In the original work $[8,14]$ convergence of solutions to a uniquely determined self-similar solution was predicted. However, it is by now well known $[5,9,11]$ that the long-time behavior of solutions depends sensitively on the initial data. In $[9,11]$ a necessary criterion for convergence to any self-similar solution which behaves like a finite power at the end of its (compact) support is given. It says that the data have to be regularly varying at the end of their support with the same power. This criterion is also shown to be sufficient if the power is sufficiently small and for data which are close to self-similar.

In this paper we extend the local stability result to the whole range of self-similar solutions with compact support. Our first main result establishes global stability of self-similar solutions with not too large power. The proof relies on a global contraction argument for the spreading of characteristics. In addition, we also establish upper and lower bounds for the coarsening rates of the system for a suitable class of initial data whose variation is bounded at the end of the support but not necessarily regular.
\end{abstract}

Key words: Kinetics of phase transitions, domain coarsening, asymptotic behavior, self-similarity, dependence on initial data, stability, bounds on coarsening rates

\section{Introduction}

The classical mean-field model by Lifshitz, Slyozov and Wagner (LSW) describes coarsening of a dilute dispersion of droplets in a conservative first

\footnotetext{
${ }^{1}$ Humboldt Universität zu Berlin, Unter den Linden 6, 10099 Berlin, Germany.

${ }^{2}$ Departamento de Matemática Aplicada, Facultad de Matemáticas, Universidad Complutense, 28040 Madrid, Spain.
} 
order phase transition $[8,14]$. The model is based on the assumption that particles interact by diffusional mass exchange with a common mean-field $\theta(t)$. This ansatz leads to the evolution law

$$
\frac{d}{d t} v=v^{1 / 3} \theta(t)-1
$$

for the volume $v=v(t)$ for a particle. Since nucleation of particles is negligible in the late stage of a phase transformation and collisions of particles can be neglected in the dilute regime, equation (1.1) implies that the particle size distribution $f=f(v, t)$ satisfies

$$
\partial_{t} f+\partial_{v}\left(\left(v^{1 / 3} \theta(t)-1\right) f\right)=0, \quad v>0, t>0
$$

where the mean-field $\theta$ is determined by the constraint that the volume fraction of particles is conserved. Together with (1.2) this is equivalent to

$$
\theta(t)=\frac{\int_{0}^{\infty} f(v, t) d v}{\int_{0}^{\infty} v^{1 / 3} f(v, t) d v} .
$$

Equation (1.2) is supplemented by the initial condition

$$
f(v, 0)=f_{0}(v),
$$

where $f_{0}$ is in general a given positive Radon measure. The goal of this paper is to characterize the self-similar large-time behavior of model (1.2)-(1.4).

In their classical theory LSW argued that for large times the size distribution behaves self-similar and that the self-similar limit is unique, smooth and has compact support. However, equations (1.2), (1.3) have in addition a whole one-parameter family of self-similar solutions, all with compact support. Those are not smooth at the end of their support but behave like a polynomial with finite power and the arguments of LSW suggest that they are unstable. Numerical simulations [5] and a rigorous mathematical analysis [9] however reveal that on the contrary the long-time behavior of solutions to (1.2), (1.3) in self-similar variables depends very sensitively on the initial data (see also [11, 2] for a related equation and [1] for a simplified LSW model). The main result in [9] gives a necessary criterion for convergence towards any of the non-smooth self-similar solutions. The condition states that the data must be regularly varying at the end of their support with the corresponding power. The conjecture in [9] is that this condition is also sufficient for convergence to any non-smooth self-similar solution. A proof is given for sufficiently small powers and data which are close in a suitable sense to the self-similar solution. 
In the present paper we improve the results of [9] in several directions. First we establish in Section 3 the local stability result for all self-similar solutions with finite power. A major new contribution, however, is a global stability result for self-similar solutions with not too large power. We show in Section 4 that any solution converges to the self-similar solution if the data are regularly varying with the same power at the end of their support. No further assumption on the data are needed for this result.

In Section 5 we also derive upper and lower bounds for the coarsening rates of a solution, even if the data are not regularly varying at the end of their support. Only recently, mathematically rigorous results on the growth rates within several coarsening systems have become available starting with the work [6] where time averaged upper bounds for coarsening within the Cahn-Hilliard theory have been derived from an energy-dissipation relation. Following the ideas of [6] similar results have been obtained for other coarsening systems $[7,3,4]$, however all of them give only time-averaged upper bounds. In general, lower bounds for coarsening rates can also not be expected, since there are initial data for which coarsening is much slower than the expected power law. To our knowledge, there is presently no work which quantitatively characterizes for some coarsening system initial data for which the upper bound is sharp.

Our approach in this paper is different from the one in [6]. We consider one of the simplest coarsening model, the mean-field theory, and derive pointwise upper and lower bounds for the coarsening rates. This naturally requires to make some assumptions on the initial data, since in general the result cannot be expected, e.g. if the initial data consist of a finite set of of Dirac measures for which coarsening stops after a finite time. Our result presents only a first step in the derivation of bounds for coarsening rates. We still need to require that the variation of the initial data at the end of the support is bounded and that this bound is sufficiently small. Besides being able to relax the latter condition it would finally be desirable to use these bounds to obtain results for the transient dynamics of a large but discrete system.

Finally we remark that in [9] no necessary (and possibly sufficient) criterion for convergence towards the smooth self-similar solution is given. In a second paper [13] we establish such a criterion with methods similar to those developed in the present paper and show local stability. It turns out that the criterion is much less stringent than the condition of regular variation. 


\section{Self-similar solutions}

We denote in the following by $\varphi=\varphi(v, t)$ the fraction of existing particle with volume larger than $v$, i.e. $\varphi(v, t)=\int_{v^{+}}^{\infty} f(y, t) d y$. Notice that with this definition $\varphi(\cdot, t)$ is right-continuous for all $t$. Then, equations (1.2), (1.3) read

$$
\begin{aligned}
\partial_{t} \varphi+\left(\theta(t) v^{1 / 3}-1\right) \partial_{v} \varphi & =0, \quad v>0, t>0 \\
\theta(t) & =\frac{\bar{\varphi}(t)}{\frac{1}{3} \int v^{-2 / 3} \varphi(v, t) d v},
\end{aligned}
$$

where $\bar{\varphi}(t)$ is the fraction of existing particles at time $t$, that is

$$
\bar{\varphi}(t)=\lim _{v \rightarrow 0, v>0} \varphi(v, t)=\varphi\left(0^{+}, t\right) .
$$

In equation (2.2) and in the following we use the abbreviation $\int d v$ to denote the integral $\int_{0^{+}}^{\infty} d v$, that is we do not include the 'dead' particles at $v=0$.

As pointed out before, the mean-field $\theta(t)$ is just such that the volume fraction of particles is preserved by the evolution (2.1)-(2.2), that is

$$
\int_{0^{+}}^{\infty} \varphi(v, t) d v=\int_{0^{+}}^{\infty} \varphi(v, 0) d v \quad \text { for all } t>0,
$$

where the initial data are given correspondingly by

$$
\varphi_{0}(v):=\int_{v^{+}}^{\infty} f_{0}(y) d y .
$$

Here, $f_{0}$ is a measure with compact support $[0, \bar{v}(0)]$, such that $\varphi_{0}$ is a left-continuous decreasing function with the same support. It has been established in [10] that for given $\varphi_{0}$ there exists a unique solution of (2.1), (2.2) (in the sense of distributions) which is continuous in time with values in the set of decreasing positive functions. The corresponding mean field $\theta$ is locally bounded. We also refer to [12] for a corresponding well-posedness theory for data with finite first moment.

Notice that when the data have compact support, then $\varphi(\cdot, t)$ has also compact support for all $t>0$ and we denote by

$$
\bar{v}(t):=\sup \{v \mid \varphi(v, t)>0\}
$$

the end of the support at time $t$. Since $\bar{v}(t)^{1 / 3} \theta(t) \geq 1$, we find $\partial_{t} \bar{v}(t) \geq 0$ for all $t$ and equality only holds if there are only particles of one size present, i.e. if $\varphi(v, t)$ is the multiple of the characteristic function of $(0, \bar{v}(t))$. 
The long-time behavior (not yet in a self-similar time scale) of solutions to (2.1), (2.2) has been characterized in [9]. Here one has to distinguish two cases. The first is that there is a positive fraction of particles which have volume $\bar{v}(0)$. In other words, $f_{0}$ carries a Dirac mass at the end of the support, or, equivalently $\varphi_{0}$ is discontinuous at the end of the support. In this case the solution converges to a Dirac mass and no self-similar behavior occurs.

Since we are interested in self-similar long-time behavior we assume from

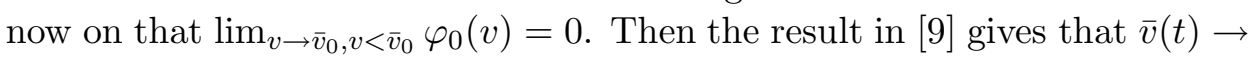
$\infty$ as $t \rightarrow \infty$ and $\lim _{t \rightarrow \infty} \bar{\varphi}(t)=0$.

Now we can introduce the following self-similar variables. We define

$$
u=\frac{v}{\bar{v}(t)}, \quad \tau=\ln \left(\frac{\bar{v}(t)}{\bar{v}(0)}\right), \quad F(u, \tau)=\bar{v}(t) \varphi(v, t)
$$

and introduce $\kappa(\tau)$ via

$$
\frac{1}{\kappa(\tau)}=\partial_{t} \bar{v}(t)=\bar{v}(t)^{1 / 3} \theta(t)-1
$$

Notice, that $\kappa(\tau)>0$ for all $\tau$, since $\partial_{t} \bar{v}(t)>0$ for all $t$. Furthermore, since $\phi_{0}$ is continuous at the end of its support, it also follows $\lim _{u \rightarrow 1} F(u, 0)=0$ and, since the evolution preserves this property, we have

$$
\lim _{u \rightarrow 1} F(u, \tau)=0
$$

for all $\tau>0$. With this change of variables we find

$$
\begin{aligned}
0 & =\partial_{t} \varphi+\left(\theta v^{1 / 3}-1\right) \partial_{v} \varphi \\
& =\frac{1}{\bar{v}^{2} \kappa}\left(-F+\partial_{\tau} F-u \partial_{u} F+\kappa\left(\theta v^{1 / 3}-1\right) \partial_{u} F\right) .
\end{aligned}
$$

Since $-u+\kappa\left(\theta v^{1 / 3}-1\right)=u^{1 / 3}-u+\kappa\left(u^{1 / 3}-1\right)$ we obtain

$$
\partial_{\tau} F+\left(u^{1 / 3}-u+\kappa(\tau)\left(u^{1 / 3}-1\right)\right) \partial_{u} F=F,
$$

where $\kappa(\tau)$ is such that

$$
\int_{0^{+}}^{1} F(u, \tau) d u=\int_{0^{+}}^{1} F(u, 0) d u \quad \text { for all } \tau>0 .
$$

Self-similar solutions of the original problem are given by stationary solutions of (2.10) and those are

$$
F_{\kappa_{*}}(u)=C \exp \left(-\int_{0}^{u} \frac{d y}{\kappa_{*}\left(1-y^{1 / 3}\right)+\left(y-y^{1 / 3}\right)} .\right)
$$


where $\kappa_{*}$ is constant and $C$ is a normalization constant. Taylor's expansion yields for $y \rightarrow 1, y<1$ that

$$
\begin{aligned}
f(y):=\kappa_{*}\left(1-y^{1 / 3}\right)+\left(y-y^{1 / 3}\right)= & \frac{\kappa_{*}-2}{3}(1-y)+\frac{\kappa_{*}+1}{9}(1-y)^{2} \\
& +\frac{5\left(\kappa_{*}+1\right)}{81}(1-y)^{3}+O\left(|1-y|^{4}\right)
\end{aligned}
$$

and we see that $F_{\kappa_{*}}$ is positive and vanishes at $u=1$ only if $\kappa_{*} \geq 2$. Furthermore, we obtain for $u \rightarrow 1$ that

$$
F_{\kappa_{*}}(u) \sim \bar{C}(1-u)^{\alpha}, \quad \text { if } \kappa_{*}>2, \quad \text { with } \alpha=\frac{3}{\kappa_{*}-2},
$$

whereas

$$
F_{\kappa_{*}}(u) \sim \bar{C}(1-u)^{5 / 3} e^{-\frac{3}{1-u}}, \quad \text { if } \kappa_{*}=2 .
$$

In order to study the large-time behavior of solutions to (2.10) it will be convenient to introduce for fixed $\kappa_{*} \geq 2$ a new variable $z$ via

$$
z=\int_{0}^{u} \frac{d y}{f(y)} .
$$

Using (2.13) we find for $u \rightarrow 1$ that

$$
z \sim\left\{\begin{array}{ccc}
\frac{3}{\kappa_{*}-2} \ln \frac{1}{1-u} & : & \kappa_{*}>2 \\
\frac{3}{1-u}+\frac{5}{3} \ln (1-u) & : & \kappa_{*}=2
\end{array} .\right.
$$

Furthermore we introduce

$$
\beta(\tau):=\kappa(\tau)-\kappa_{*}
$$

and $G(z, \tau):=F(u, \tau)$ such that

$$
\partial_{\tau} G+(-1-\beta(\tau) H(z)) \partial_{z} G=G,
$$

and

$$
\int_{0^{+}}^{1} G(z, \tau) d u=\int_{0^{+}}^{1} G(z, 0) d u
$$

where

$$
H(z)=\frac{1-u^{1 / 3}}{\left(u-u^{1 / 3}\right)+\kappa_{*}\left(1-u^{1 / 3}\right)}=\frac{1}{\kappa_{*}-u^{1 / 3}\left(u^{1 / 3}+1\right)} .
$$


In view of $(2.9)$ it also follows that

$$
\lim _{z \rightarrow \infty} G(z, \tau)=0
$$

We include a brief discussion of the function $H$. We find

$$
H^{\prime}(z)=H(z)\left(1-u^{1 / 3}\right)\left(\frac{1}{3} u^{-2 / 3}+\frac{2}{3} u^{-1 / 3}\right) \geq 0
$$

and since $H(0)=\frac{1}{\kappa_{*}}$ and $\lim _{z \rightarrow \infty} H(z)=\frac{1}{\kappa_{*}-2}$ it follows

$$
\frac{1}{\kappa_{*}} \leq H(z)<\frac{1}{\kappa_{*}-2} \quad \text { for all } z<\infty .
$$

With (2.13) and (2.17) we find for $u \rightarrow 1$ (resp. $z \rightarrow \infty$ ) that

$$
H(z) \sim\left\{\begin{array}{clc}
\frac{1}{\kappa_{*}-2+\frac{\kappa_{*}+1}{3}(1-u)} \sim \frac{1}{\kappa_{*}-2+\frac{\kappa_{*}+1}{3} e^{-\frac{\kappa_{*}-2}{3} z}} & : \quad \kappa_{*}>2 \\
\frac{1}{1-u}+\frac{5}{9} \ln (1-u) \sim \frac{z}{3}+\frac{5}{9} \ln z & : \quad \kappa_{*}=2
\end{array}\right.
$$

and

$$
H^{\prime}(z) \sim\left\{\begin{array}{cc}
\left.\frac{1}{3\left(\kappa_{*}-2\right)}(1-u) \sim \frac{1}{3\left(\kappa_{*}-2\right)} e^{-\frac{\kappa_{*}-2}{3} z}\right) & : \\
\frac{1}{3} & \kappa_{*}>2 \\
: & \kappa_{*}=2
\end{array},\right.
$$

whereas for $u \rightarrow 0$ we have

$$
H^{\prime}(z) \sim \frac{1}{3 \kappa_{*}} u^{-2 / 3} .
$$

The advantage of the new variable $z$ given by (2.16) is that now selfsimilar solutions can be written in a particularly simple way, namely as $G_{\kappa_{*}}(z)=C e^{-z}$. In the following we normalize the volume fraction of particles (cf. (2.20)) such that

$$
\int_{0^{+}}^{1} G(z, \tau) d u=\int_{0^{+}}^{1} G_{0}(z) d u=1 .
$$

Then the corresponding self-similar solution for given $\kappa_{*} \geq 2$ is

$$
G_{\kappa_{*}}(z)=C_{*} e^{-z}
$$

with

$$
C_{*}=C\left(\kappa_{*}\right)=\frac{1}{\int_{0}^{1} e^{-z} d u} .
$$

Notice that $C_{*}$ is decreasing as $\kappa_{*}$ in increasing due to (2.16).

The major difference between the cases $\kappa_{*}>2$ and $\kappa_{*}=2$ is the behavior of the function $H(z)$ as $z \rightarrow \infty$. If $\kappa_{*}>2$, then $H$ is bounded, whereas if $\kappa_{*}=2$ it grows linearly. 


\section{Local stability}

In this section we establish for initial data which are close to a self-similar solution and which are regularly varying at the end of their support that the corresponding solution converges toward the self-similar profile.

\subsection{Asymptotically slowly varying functions}

We say that a function $S \in L^{\infty}(I)$ is asymptotically slowly varying at infinity, if

$$
\lim _{y \rightarrow \infty} \frac{S(y+z)}{S(y)}=1 \quad \text { locally uniformly on } \mathbb{R}_{+} .
$$

In order to define perturbations of stationary solutions which are small in an appropriate sense we introduce the following measure of the degree of oscillations of a function $S \in L^{\infty}(I)$, where $I$ is any interval in $\mathbb{R}_{+}$. We define the flatness modulus of $S$ as

$$
\bar{\omega}\left(S, z_{0}\right):=\sup _{z \geq z_{0}} \sup _{y \in[z, z+1]} \frac{|S(z)-S(y)|}{|S(z)|} .
$$

It is easily established that $S$ is asymptotically slowly varying at infinity if and only if

$$
\lim _{z_{0} \rightarrow \infty} \bar{\omega}\left(S, z_{0}\right)=0 .
$$

Notice that our definitions coincide with the ones used in [9]. The notation is slightly different since in [9] instead of $S$ an equivalent of $\ln S$ is considered.

\subsection{A necessary condition for convergence}

The following result has already been proved in [9]. For the sake of completeness we present the proof, in which we will also introduce notation and estimates needed in subsequent proofs.

Theorem 3.1. We consider a fixed but arbitrary $\kappa_{*}>2$. Let $G(z, \tau)$ the solution of (2.19) with data $G_{0}(z)$.

Suppose that

$$
\lim _{\tau \rightarrow \infty} G(z, \tau)=G_{\kappa_{*}}(z)=C_{*} e^{-z}
$$

locally uniformly on $\mathbb{R}_{+}$. Then the function $S_{0}(z):=G_{0}(z) e^{z}$ is asymptotically slowly varying as $z \rightarrow \infty$. 
Proof. We first notice that (2.2), (2.7), (2.8) and (2.29) imply

$$
\frac{1}{\kappa_{*}}=-1+\frac{3}{\int_{0}^{1} u^{-2 / 3} e^{-z} d u}
$$

We are going to show the following formula for $\beta(\tau)$ :

$$
\beta(\tau)=\frac{\kappa_{*}\left(\kappa_{*}+1\right)^{2}}{9} \frac{\int_{0}^{1} u^{-2 / 3}\left(\frac{G(z, \tau)}{G\left(0^{+}, \tau\right)}-e^{-z}\right) d u}{\kappa_{*}-\frac{\kappa_{*}-2}{3} \frac{\kappa_{*}+1}{3} \int_{0}^{1} u^{-2 / 3}\left(\frac{G(z, \tau)}{G\left(0^{+}, \tau\right)}-e^{-z}\right) d u} .
$$

As a consequence of (3.4) and (3.5) we find

$$
\lim _{\tau \rightarrow 0} \beta(\tau)=0 .
$$

We denote by $\tilde{z}\left(\tau, z_{0}\right)$ the characteristic curve corresponding to (2.19). More precisely, we define

$$
\frac{\partial \tilde{z}}{\partial \tau}=-1-\beta(\tau) H(\tilde{z}), \quad \tilde{z}\left(0, z_{0}\right)=z_{0},
$$

such that

$$
\frac{\partial \tilde{z}}{\partial z_{0}}=\exp \left(-\int_{0}^{\tau} \beta(s) H^{\prime}\left(\tilde{z}\left(s, z_{0}\right)\right) d s\right) .
$$

By integrating (2.19) along characteristics we find that

$$
G\left(\tilde{z}\left(\tau, z_{0}\right), \tau\right)=e^{\tau} G_{0}\left(z_{0}\right) .
$$

Let us denote by $\bar{z}_{0}=\bar{z}_{0}(\tau)$ the initial value of the characteristic which reaches $z=0$ at time $\tau$, i.e. $\tilde{z}\left(\tau, \bar{z}_{0}(\tau)\right)=0$.

From the assumption that $G(z, \tau) \rightarrow G_{\kappa_{*}}(z)$ and (3.6), (3.7) and (3.9) it follows that $\lim _{\tau \rightarrow \infty} \bar{z}_{0}(\tau)=\infty$ and $\lim _{z_{0} \rightarrow \infty} G_{0}\left(z_{0}\right)=0$. Furthermore, (3.9) yields

$$
\frac{G(z, \tau)}{G\left(0^{+}, \tau\right)}=\frac{G_{0}\left(z_{0}\right)}{G_{0}\left(\bar{z}_{0}\right)}
$$

Together with the assumption of convergence (3.4) this implies

$$
\lim _{\tau \rightarrow \infty} \frac{G(z, \tau)}{G\left(0^{+}, \tau\right)}=\lim _{\tau \rightarrow \infty} \frac{G_{0}\left(\bar{z}_{0}+\left(z_{0}-\bar{z}_{0}\right)\right)}{G_{0}\left(\bar{z}_{0}\right)}=e^{-z}
$$

uniformly in compact sets for $z_{0} \geq \bar{z}_{0}$. If we fix $z$ and show that

$$
\lim _{\tau \rightarrow \infty}\left(z_{0}-\bar{z}_{0}-\tilde{z}\left(\tau, z_{0}\right)\right)=0 .
$$


then (3.11) is equivalent to

$$
\lim _{\bar{z}_{0} \rightarrow \infty} \frac{S_{0}\left(\bar{z}_{0}+z\right)}{S_{0}\left(\bar{z}_{0}\right)}=1
$$

which just means that $S$ is asymptotically slowly varying.

Thus, we are now going to show (3.12). Fix $z=\tilde{z}\left(\tau, z_{0}\right)$. For given $\varepsilon>0$ there exists due to (3.6) a $\tau_{\varepsilon}$ such that $|\beta(\tau)| \leq \varepsilon$ for all $\tau \geq \tau_{\varepsilon}$. For $s \in\left[\tau_{\varepsilon}, \tau\right]$ we find $\tilde{z}^{\prime} \leq-\frac{1}{2}$ and hence

$$
\tilde{z}\left(s, z_{0}\right) \geq z+\frac{1}{2}(\tau-s) \quad \text { for } s \in\left[\tau_{\varepsilon}, \tau\right] .
$$

In particular $\tilde{z}\left(\tau_{\varepsilon}, z_{0}\right) \geq z+\frac{1}{2}\left(\tau-\tau_{\varepsilon}\right)$. Since $\beta$ and $H$ are bounded, we also find

$$
\tilde{z}\left(s, z_{0}\right) \geq z+\frac{1}{2} \tau-C_{\varepsilon}
$$

for all $s \in\left[0, \tau_{\varepsilon}\right]$.

On the other hand, due to the exponential decay of $H^{\prime}$ (cf. (2.26)), we find a value $\hat{z} \geq z$ such that $H^{\prime}(\tilde{z}) \leq e^{-\delta \tilde{z}}$ for $\tilde{z} \geq \hat{z}$ and some $\delta>0$. From (3.13) we know that $\tilde{z}\left(s, z_{0}\right) \geq \hat{z}$ for all $\tau_{\varepsilon} \leq s \leq \tau-2(\hat{z}-z)$. Hence we can conclude, recalling (3.14), that

$$
\begin{aligned}
\mid \int_{0}^{\tau} \beta(s) & H^{\prime}\left(\tilde{z}\left(s, z_{0}\right)\right) d s\left|\leq \int_{\tau-2(\hat{z}-z)}^{\tau}\right| \beta(s) H^{\prime}\left(\tilde{z}\left(s, z_{0}\right)\right) \mid d s \\
& +\int_{\tau_{\varepsilon}}^{\tau-2(\hat{z}-z)}\left|\beta(s) H^{\prime}\left(\tilde{z}\left(s, z_{0}\right)\right)\right| d s+\int_{0}^{\tau_{\varepsilon}}\left|\beta(s) H^{\prime}\left(\tilde{z}\left(s, z_{0}\right)\right)\right| d s \\
& \leq C(\hat{z}-z) \varepsilon+\frac{\varepsilon}{\delta} e^{-\delta \tau_{\varepsilon}}+C(z, \varepsilon) e^{-\delta \tau} \\
& \leq C \varepsilon+C_{\varepsilon} e^{-\delta \tau}
\end{aligned}
$$

whence $\lim \sup _{\tau \rightarrow \infty}\left|\int_{0}^{\tau} \beta(s) H^{\prime}\left(\tilde{z}\left(s, z_{0}\right)\right) d s\right|=0$ and as a consequence

$$
\lim _{\tau \rightarrow \infty} \exp \left(-\int_{0}^{\tau} \beta(s) H^{\prime}\left(\tilde{z}\left(s, z_{0}\right)\right) d s\right)=1 .
$$

Identity (3.12) follows now from (3.8) and (3.15).

To finish the proof of the theorem it remains to derive (3.5). We conclude from (2.2) and the definitions (2.7), (2.8) and (2.29) that

$$
\frac{3}{\int_{0}^{1} u^{-2 / 3} \frac{G(z, \tau)}{G\left(0^{+}, \tau\right)} d u}-\frac{3}{\int_{0}^{1} u^{-2 / 3} e^{-z} d u}=\frac{1}{\kappa(\tau)}-\frac{1}{\kappa_{*}}=-\frac{\beta(\tau)}{\kappa_{*}\left(\kappa_{*}+\beta(\tau)\right)} .
$$


This implies

$$
\begin{aligned}
& \frac{\beta(\tau)}{\kappa_{*}\left(\kappa_{*}+\beta(\tau)\right)}=\frac{\int_{0}^{1} u^{-2 / 3}\left(\frac{G(z, \tau)}{G\left(0^{+}, \tau\right)}-e^{-z}\right) d u}{\left(\int_{0}^{1} u^{-2 / 3} \frac{G(z, \tau)}{G\left(0^{+}, \tau\right)} d u\right)\left(\int_{0}^{1} u^{-2 / 3} e^{-z} d u\right)} \\
& =\frac{\int_{0}^{1} u^{-2 / 3}\left(\frac{G(z, \tau)}{G\left(0^{+}, \tau\right)}-e^{-z}\right) d u}{\left(\int_{0}^{1} u^{-2 / 3} e^{-z} d u\right)\left(\int_{0}^{1} u^{-2 / 3}\left(\frac{G(z, \tau)}{G\left(0^{+}, \tau\right)}-e^{-z}\right) d u+\int_{0}^{1} u^{-2 / 3} e^{-z} d u\right)}
\end{aligned}
$$

On the other hand we have

$$
\int_{0}^{1} u^{-2 / 3} e^{-z} d u=\frac{3 \kappa_{*}}{\kappa_{*}+1}
$$

which gives with (3.16) that

$$
\frac{\beta(\tau)}{\kappa_{*}+\beta(\tau)}=\frac{\left(\kappa_{*}+1\right)^{2}}{9 \kappa_{*}} \frac{\int_{0}^{1} u^{-2 / 3}\left(\frac{G(z, \tau)}{G\left(0^{+}, \tau\right)}-e^{-z}\right) d u}{\left(\frac{\kappa_{*}+1}{3 \kappa_{*}} \int_{0}^{1} u^{-2 / 3}\left(\frac{G(z, \tau)}{G\left(0^{+}, \tau\right)}-e^{-z}\right) d u+1\right)}
$$

Solving the last identity for $\beta$ finally yields (3.5).

\subsection{A locally sufficient condition for convergence}

We are now going to establish that self-similar solutions are asymptotically stable with respect to small perturbations (defined in an appropriate sense). This has been shown for sufficiently large $\kappa_{*}$ in [9]. Here we show the corresponding result for all $\kappa_{*}>2$.

Theorem 3.2. Suppose that $\kappa_{*}>2$ and let $C_{*} e^{-z}$ be the corresponding selfsimilar solution. Let us further suppose that $S_{0}\left(z_{0}\right)=G_{0}\left(z_{0}\right) e^{z_{0}}$ satisfies for sufficiently small $\varepsilon_{0}=\varepsilon_{0}\left(\kappa_{*}\right)$ that

$$
\bar{\omega}\left(S_{0}, 0\right) \leq \varepsilon_{0}
$$

and

$$
\lim _{z_{0} \rightarrow \infty} \bar{\omega}\left(S_{0}, z_{0}\right)=0 .
$$

Then

$$
\lim _{\tau \rightarrow \infty} G(z, \tau)=C_{*} e^{-z}
$$

locally uniformly on $\mathbb{R}^{+}$. 
Proof. With $S(z, \tau):=G(z, \tau) e^{z}$ we find, using (2.19), that

$$
\begin{aligned}
\partial_{\tau} S-(1+\beta(\tau) H(z)) \partial_{z} S & =-\beta(\tau) H(z) S, \\
S(z, 0) & =S_{0}(z) .
\end{aligned}
$$

We also rewrite (3.5) in terms of $S$ which yields

$$
\beta(\tau)=\frac{\kappa_{*}\left(\kappa_{*}+1\right)^{2}}{9} \frac{\int_{0}^{1} u^{-2 / 3}\left(\frac{S(z, \tau)}{S\left(0^{+}, \tau\right)}-1\right) e^{-z} d u}{\kappa_{*}-\frac{\kappa_{*}-2}{3} \frac{\kappa_{*}+1}{3} \int_{0}^{1} u^{-2 / 3}\left(\frac{S(z, \tau)}{S\left(0^{+}, \tau\right)}-1\right) e^{-z} d u} .
$$

Integrating (3.20) along characteristics we obtain

$$
\frac{S(z, \tau)}{S\left(0^{+}, \tau\right)}=\frac{S_{0}\left(z_{0}\right)}{S_{0}\left(\bar{z}_{0}(\tau)\right)} \exp \left(-\int_{0}^{\tau} \beta(s)\left\{H\left(\tilde{z}\left(s, z_{0}\right)\right)-H\left(\tilde{z}\left(s, \bar{z}_{0}(\tau)\right)\right)\right\} d s\right),
$$

where $z=\tilde{z}\left(\tau, z_{0}\right)$ and $\tilde{z}\left(\tau, \bar{z}_{0}(\tau)\right)=0$. Therefore

$$
\begin{aligned}
& \int_{0}^{1} u^{-2 / 3}\left(\frac{S(z, \tau)}{S\left(0^{+}, \tau\right)}-1\right) e^{-z} d u \\
& =\int_{0}^{1} u^{-2 / 3} e^{-z}\left(\frac{S_{0}\left(z_{0}\right)}{S_{0}\left(\bar{z}_{0}\right)}-1\right) \\
& \quad \cdot \exp \left(-\int_{0}^{\tau} \beta(s)\left(H\left(\tilde{z}\left(s, z_{0}\right)\right)-H\left(\tilde{z}\left(s, \bar{z}_{0}\right)\right)\right) d s\right) d u \\
& +\int_{0}^{1} u^{-2 / 3} e^{-z}\left(\exp \left(-\int_{0}^{\tau} \beta(s)\left(H\left(\tilde{z}\left(s, z_{0}\right)\right)-H\left(\tilde{z}\left(s, \bar{z}_{0}\right)\right)\right) d s\right)-1\right) d u \\
& =: I_{1}+I_{2} .
\end{aligned}
$$

We are going to show that $I_{1}$ is small due to the assumption (3.17). The second term $I_{2}$ we will linearize to obtain an approximate integral equation for $\beta(\tau)$.

Define $\delta(\tau):=\sup _{0<s<\tau}|\beta(s)|$ and suppose that

$$
\delta(\tau) \leq \min \left(\delta_{0}\left(\kappa_{*}\right), \frac{\kappa_{*}-2}{2}\right) \quad \text { for } \tau \in\left[0, \tau_{0}\right]
$$

for some sufficiently small $\delta_{0}>0$. In view of (2.24) we have for those $\tau$ that $(1+\beta(\tau) H(z))>\frac{1}{2}$, and thus $\tilde{z}\left(\tau, z_{0}\right)$ decreases linearly for $\tau \in\left[0, \tau_{0}\right]$. We are going to argue that

$$
0<\frac{1}{C\left(\kappa_{*}\right)} \leq \frac{\partial \tilde{z}}{\partial z_{0}} \leq C\left(\kappa_{*}\right) \quad \text { for } \tau \in\left[0, \tau_{0}\right]
$$


uniformly in $\tau_{0}$ and $z_{0}$. Recall that $H^{\prime}(z) \leq e^{-\delta z}$ for $z \geq \hat{z}$. Furthermore we have $\tilde{z}\left(s, z_{0}\right) \geq z+\frac{1}{2}(\tau-s)$ for all $\tau \in\left[0, \tau_{0}\right], s \in[0, \tau]$ and thus $\tilde{z}\left(s, z_{0}\right) \geq \hat{z}$ for $s \in[0, \tau-2(\hat{z}-z)]$. Now (3.25) follows from (3.8) analogously to the proof of (3.15).

To find a bound in (3.23) we first need to show that the exponential term in $I_{1}$ is uniformly bounded in $\tau_{0}$. However, if $\tau_{0} \rightarrow \infty$ we can argue again as in (3.15), using the exponential decay of $\left|H\left(\tilde{z}\left(s, z_{0}\right)\right)-H\left(\tilde{z}\left(s, \bar{z}_{0}\right)\right)\right|$ as $\tau-s \rightarrow \infty$, to conclude that

$$
\exp \left(-\int_{0}^{\tau} \beta(s)\left(H\left(\tilde{z}\left(s, z_{0}\right)\right)-H\left(\tilde{z}\left(s, \bar{z}_{0}\right)\right)\right) d s\right) \leq C\left(\kappa_{*}\right)
$$

and thus

$$
\left|I_{1}\right| \leq C\left(\kappa_{*}\right) \int_{0}^{1} u^{-2 / 3} e^{-z}\left|\frac{S_{0}\left(z_{0}\right)}{S_{0}\left(\bar{z}_{0}\right)}-1\right| d u .
$$

With the definition of $\bar{\omega}\left(\cdot, z_{0}\right)$ we find

$$
\left|\frac{S_{0}\left(z_{0}\right)}{S_{0}\left(\bar{z}_{0}\right)}-1\right| \leq C\left|z_{0}-\bar{z}_{0}\right| \bar{\omega}\left(S_{0}, z_{0}\right)
$$

On the other hand, estimate (3.25) implies that $\left|z_{0}-\bar{z}_{0}\right| \leq C\left(\kappa_{*}\right) z$ and hence

$$
\left|I_{1}\right| \leq C\left(\kappa_{*}\right) \bar{\omega}\left(S_{0}, \bar{z}_{0}\right) \int_{0}^{1} u^{-2 / 3} e^{-z} z d u \leq C\left(\kappa_{*}\right) \bar{\omega}\left(S_{0}, \bar{z}_{0}\right)
$$

for all $\tau \in\left[0, \tau_{0}\right]$.

Next, we have to find a suitable approximation for $I_{2}$. Once more we can use the exponential decay of $H^{\prime}(z)$ as $z \rightarrow \infty$ to conclude that

$$
\int_{0}^{\tau}\left|H\left(\tilde{z}\left(s, z_{0}\right)\right)-H\left(\tilde{z}\left(s, \bar{z}_{0}\right)\right)\right| d s \leq C\left(\kappa_{*}\right) \quad \text { for } \tau \in\left[0, \tau_{0}\right]
$$

uniformly in $\tau_{0} \rightarrow \infty$. Hence, if $\delta_{0}\left(\kappa_{*}\right)$ in (3.24) is sufficiently small, we can use Taylor's expansion to obtain

$$
I_{2}=-\int_{0}^{\tau} \beta(s)\left(\int_{0}^{1} u^{-2 / 3} e^{-z}\left(H\left(\tilde{z}\left(s, z_{0}\right)\right)-H\left(\tilde{z}\left(s, \bar{z}_{0}\right)\right)\right) d u\right) d s+O\left(\delta(\tau)^{2}\right) .
$$

We write

$$
\begin{aligned}
& H\left(\tilde{z}\left(s, z_{0}\right)\right)-H\left(\tilde{z}\left(s, \bar{z}_{0}\right)\right) \\
& \quad(H(z+(\tau-s))-H(\tau-s)) \\
& \quad+\left(H\left(\tilde{z}\left(s, z_{0}\right)\right)-H(z+(\tau-s))\right)-\left(H\left(\tilde{z}\left(s, \bar{z}_{0}\right)\right)-H(\tau-s)\right) .
\end{aligned}
$$


Using equation (3.7) for the characteristics it follows that

$$
\left|\tilde{z}\left(s, z_{0}\right)-(z+(\tau-s))\right|+\left|\tilde{z}\left(s, \bar{z}_{0}(\tau)\right)-(\tau-s)\right| \leq C \delta(\tau)|\tau-s| .
$$

Again, the exponential decay of $H^{\prime}(z)$ as $z \rightarrow \infty$ and the Hölder regularity at $z=0$ (cf. (2.27)), imply that

$$
\left|H\left(z_{1}\right)-H\left(z_{2}\right)\right| \leq C e^{-\gamma \min \left(z_{1}, z_{2}\right)}\left|z_{1}-z_{2}\right|^{1 / 3}
$$

for some $\gamma>0$. Hence

$$
\begin{aligned}
& \left|\int_{0}^{\tau} \beta(s) \int_{0}^{1} u^{-2 / 3} e^{-z}\left(H\left(\tilde{z}\left(s, z_{0}\right)\right)-H(z+(\tau-s))\right) d u d s\right| \\
& \leq C \delta(\tau)^{4 / 3}\left|\int_{0}^{\tau} \int_{0}^{1} u^{-2 / 3} e^{-z} e^{-\gamma \min \left(\tilde{z}\left(s, z_{0}\right), z+\tau-s\right)}\right| \tau-s|d u d s| \\
& \leq C \delta(\tau)^{4 / 3}
\end{aligned}
$$

Similarly we find

$$
\left|\int_{0}^{1} u^{-2 / 3} e^{-z}\left(H\left(\tilde{z}\left(s, \bar{z}_{0}\right)\right)-H(\tau-s)\right) d u d s\right| \leq C \delta(\tau)^{4 / 3} .
$$

Thus

$$
\begin{gathered}
I_{2}=-\int_{0}^{\tau} \beta(s) K(\tau-s) d s+O\left(\delta(\tau)^{4 / 3}\right) \\
K(\tau-s)=\int_{0}^{1} u^{-2 / 3} e^{-z}(H(z+(\tau-s))-H(\tau-s)) d u .
\end{gathered}
$$

Combining (3.23), (3.26), (3.27) and (3.28) we can rewrite equation (3.21), as long as (3.24) holds, as

$$
\beta(\tau)=-\frac{\left(\kappa_{*}+1\right)^{2}}{9} \int_{0}^{\tau} \beta(s) K(\tau-s) d s+O\left(\delta(\tau)^{4 / 3}\right)+O\left(\bar{\omega}\left(S_{0}, z_{0}\right)\right) .
$$

The derivation of formula (3.29) is the key step of the proof. It reduces the problem of stability within the LSW model to the proof of stability of an integral equation.

Lemma 3.3. Suppose that $G: \mathbb{R}_{+} \rightarrow \mathbb{R}$ is a nonnegative decreasing function which satisfies for some $\theta>0$ that

$$
G^{\prime}(\tau) \leq-\theta G(\tau), \quad \text { for all } \tau>0 .
$$


Suppose further that $f: \mathbb{R}_{+} \rightarrow \mathbb{R}$ is a continuous function which satisfies

$$
f(\tau)=-\int_{0}^{\tau} G(\tau-s) f(s) d s+\lambda(\tau) .
$$

Then

$$
|f(\tau)| \leq C\left(|\lambda(\tau)|+\int_{0}^{\tau} e^{-\gamma(\tau-s)}|\lambda(s)| d s\right)
$$

for some $\gamma>0$.

The proof of Lemma 3.3 is given in the Appendix. We now show how to use it in Theorem 3.2. First we notice that the kernel $K$ satisfies the assumptions of the Lemma. Indeed, $K$ is positive, since $H$ is monotonically increasing (cf. (2.23)). Furthermore we compute

$$
H^{\prime \prime}(z)=\frac{H(z)}{9}\left\{\left(1-u^{1 / 3}\right)^{2}\left(2 u^{-1 / 3}+u^{-2 / 3}\right)^{2}-f(u)\left(u^{-4 / 3}+2 u^{-5 / 3}\right)\right\}
$$

and one easily checks that $H^{\prime \prime}(z)<0$ for all $z \in(0, \infty)$ and $\kappa_{*} \geq 2$. This implies that $K^{\prime}(\tau) \leq 0$. To establish (3.30) we notice that both, $H^{\prime}$ and $H^{\prime \prime}$, are proportional to $e^{-\frac{\kappa *-2}{3} z}$ as $z \rightarrow \infty$ which implies (3.30) for $K$.

Then we use a standard continuation argument. If $\bar{\omega}\left(S_{0}, 0\right)$ is sufficiently small, then (3.29) implies that (3.24) holds for $\tau=0$. Lemma 3.3 then implies the estimate

$$
|\beta(\tau)| \leq C \delta(\tau)^{4 / 3}+C \bar{\omega}\left(S_{0}, \bar{z}_{0}\right),
$$

which is satisfied as long as (3.24) is valid. This inequality yields

$$
|\delta(\tau)| \leq C \bar{\omega}\left(S_{0}, \bar{z}_{0}\right) .
$$

Hence, if $\bar{\omega}\left(S_{0}, 0\right)$ is sufficiently small, (3.24) will hold for any $\tau>0$.

Finally, we need to show that $\lim _{\tau \rightarrow \infty} \beta(\tau)=0$. We notice that the arguments leading to (3.29) also give

$$
\begin{aligned}
\beta(\tau)= & -\frac{\left(\kappa_{*}+1\right)^{2}}{9} \int_{0}^{\tau} \beta(s) K(\tau-s) d x \\
& +O\left(\sup _{\tau}|\delta(\tau)|^{1 / 3} \int_{0}^{\tau}|\beta(s)| e^{-\theta(\tau-s)} d s\right)+O\left(\bar{\omega}\left(S_{0}, \bar{z}_{0}\right)\right) .
\end{aligned}
$$

Using again Lemma 3.3 it follows that

$$
\sup _{s \in[\tau-n, \tau]}|\beta(s)| \leq C\left(\bar{\omega}\left(S_{0}, \bar{z}_{0}\right)+\sup _{\tau}\left(|\delta(\tau)|^{4 / 3}\right) e^{-\theta n}\right)
$$


from which we conclude, using (3.18), that

$$
\limsup _{\tau \rightarrow \infty}|\beta(\tau)| \leq C \sup _{\tau}\left(|\delta|^{4 / 3}\right) e^{-\theta n}
$$

for any $n>0$, which implies that $\lim _{\tau \rightarrow \infty} \beta(\tau)=0$.

We can now deduce from (3.22) that

$$
\lim _{\tau \rightarrow \infty} \frac{S(z, \tau)}{S\left(0^{+}, \tau\right)}=\lim _{\tau \rightarrow \infty} \frac{S_{0}\left(z_{0}\right)}{S_{0}\left(\bar{z}_{0}(\tau)\right)}
$$

for all $z$. On the other hand, (3.18) and (3.12) (which follows from $\beta(\tau) \rightarrow$ 0 ) imply that $\lim _{\tau \rightarrow \infty} \frac{S_{0}\left(z_{0}\right)}{S_{0}\left(\bar{z}_{0}(\tau)\right)}=1$. Hence, we can deduce the desired convergence (3.19) from (3.33), which completes the proof of the theorem.

\section{Global stability}

In this section we show that assumption (3.17) in Theorem 3.2 can be dropped if $\kappa_{*}$ is sufficiently large. Therefore, in this case every solution of the LSW system which behaves locally as a self-similar solution near the maximum radius converges to the self-similar solution.

Theorem 4.1. There exists a $\kappa_{0}>2$ such that for any $\kappa_{*}>\kappa_{0}$ the following is true. If $S_{0}\left(z_{0}\right)=G_{0}\left(z_{0}\right) e^{z_{0}}$ satisfies

$$
\lim _{z_{0} \rightarrow \infty} \varpi\left(S_{0}, z_{0}\right)=0
$$

then

$$
\lim _{\tau \rightarrow \infty} \frac{G(z, \tau)}{G\left(0^{+}, \tau\right)}=C_{*} e^{-z}
$$

locally uniformly on $\mathbb{R}^{+}$.

We decompose the proof of Theorem 4.1 into a sequence of Lemmas.

Lemma 4.2. We have

$$
\lim _{\tau \rightarrow \infty} \bar{z}_{0}(\tau)=\infty
$$


Proof. We first claim that

$$
\frac{\partial \tilde{z}}{\partial z_{0}} \geq \frac{\kappa_{*}-2}{\kappa_{*}}
$$

To that aim we differentiate (3.7) which yields

$$
\frac{\partial}{\partial \tau} \frac{\partial \tilde{z}}{\partial z_{0}}=-\beta(\tau) H^{\prime}(z) \frac{\partial \tilde{z}}{\partial z_{0}} .
$$

Eliminating $\beta(\tau)$ in (3.7) by using (4.5) gives

$$
\frac{H^{\prime}(z)}{H(z)} \frac{\partial \tilde{z}}{\partial \tau}-\frac{\partial}{\partial \tau}\left(\ln \left(\frac{\partial \tilde{z}}{\partial z_{0}}\right)\right)=-\frac{H^{\prime}(z)}{H(z)}
$$

which we integrate to obtain

$$
\frac{\partial \tilde{z}}{\partial z_{0}}=\frac{H(z)}{H\left(z_{0}\right)} \exp \left(\int_{0}^{\tau} \frac{H^{\prime}\left(\tilde{z}\left(s, z_{0}\right)\right)}{H\left(\tilde{z}\left(s, z_{0}\right)\right)} d s\right) .
$$

Since $H(z)$ is increasing for $z>0$ it follows $\frac{\partial \tilde{z}}{\partial z_{0}} \geq \frac{H(0)}{H(\infty)}=\frac{\kappa_{*}-2}{\kappa_{*}}$ which implies (4.4).

From (4.4) we deduce that

$$
\tilde{z}_{0}(1, \tau)-\bar{z}_{0}(\tau) \leq \frac{\kappa_{*}}{\kappa_{*}-2} .
$$

Since $\bar{z}_{0}(\tau)$ is increasing, we know that $\lim _{\tau \rightarrow \infty} \bar{z}_{0}(\tau) \leq \infty$ exists. Let us assume that $l:=\lim _{\tau \rightarrow \infty} \bar{z}_{0}(\tau)<\infty$. Then, due to (4.7) we also have $\bar{l}:=\lim \sup _{\tau \rightarrow \infty} \tilde{z}_{0}(1, \tau)<\infty$ and it follows for $z \in(0,1)$ that $G(z, \tau) \geq$ $G(1, \tau)=G_{0}\left(\tilde{z}_{0}(1, \tau)\right) e^{\tau} \geq G_{0}(\bar{l}) e^{\tau} \rightarrow \infty$ as $\tau \rightarrow \infty$. Then, however

$$
\int_{0^{+}}^{1} G(z, \tau) d u \geq G(1, \tau) \rightarrow \infty \quad \text { as } \tau \rightarrow \infty,
$$

which contradicts volume conservation (2.20).

Next we show that, in some suitable average sense, $\left(\ln G_{0}(z)\right)^{\prime} \sim-1$ as $z \rightarrow \infty$.

Lemma 4.3. Let us define $Q_{0}(z):=\left(\log \left(G_{0}(z)\right)\right)^{\prime}$ where the derivative is understood in the distributional sense. Suppose that (4.1) is satisfied. Then for any fixed $b>0$ we have

$$
\lim _{z_{0} \rightarrow \infty}\left(\sup _{\left|z_{1}-z_{0}\right| \leq b}\left\{\int_{z_{0}}^{z_{1}} Q_{0}(\xi) d \xi+\left(z_{1}-z_{0}\right)\right\}\right)=0
$$


Proof. Notice that

$$
\int_{z_{0}}^{z_{0}+b} Q_{0}(\xi) d \xi=\log \left(\frac{G_{0}\left(z_{0}+b\right)}{G_{0}\left(z_{0}\right)}\right)
$$

On the other hand (4.1) implies

$$
\frac{G_{0}\left(z_{0}+b\right)}{G_{0}\left(z_{0}\right)}=e^{-b} \frac{S_{0}\left(z_{0}+b\right)}{S_{0}\left(z_{0}\right)} \rightarrow e^{-b}
$$

as $z_{0} \rightarrow \infty$, whence the result follows.

We will use the following two auxiliary results

Lemma 4.4. Suppose that $\kappa_{*}>4$. Then

$$
\begin{aligned}
\int_{0}^{1} u^{-2 / 3} & \exp \left(-\int_{0}^{z} \frac{H(\infty)}{H(\xi)} d \xi\right) d u \\
& >\int_{0}^{1} u^{-2 / 3} \exp \left(-\int_{0}^{z} \frac{d \xi}{1-\left(\kappa_{*}-2\right) H(\xi)}\right) d u
\end{aligned}
$$

where $H$ is as in (2.21).

Proof. It follows from (2.24) that for $\kappa_{*}>4$

$$
1<2\left(\kappa_{*}-2\right) H(\xi) \quad, \quad \xi \geq 0
$$

Then $\frac{H(\infty)}{H(\xi)}=\frac{1}{\left(\kappa_{*}-2\right)} \frac{1}{H(\xi)}<\frac{1}{1-\left(\kappa_{*}-2\right) H(\xi)}$ for $\xi \geq 0$ whence (4.8) follows.

Lemma 4.5. Let us define

$$
\Phi(x):=\frac{\kappa_{*}\left(\kappa_{*}+1\right)^{2}}{9} \frac{x}{\kappa_{*}-\frac{\kappa_{*}-2}{3} \frac{\kappa_{*}+1}{3} x}
$$

Then, the following identity is satisfied

$$
-\left(\kappa_{*}-2\right)=\Phi\left(\int_{0}^{1} u^{-2 / 3}\left(\exp \left(-\int_{0}^{z} \frac{d \xi}{1-\left(\kappa_{*}-2\right) H(\xi)}\right)-e^{-z}\right) d u\right)
$$


Proof. Notice that (3.5) implies

$$
\beta(\tau)=\Phi\left(\int_{0}^{1} u^{-2 / 3}\left(\frac{G(z, \tau)}{G\left(0^{+}, \tau\right)}-e^{-z}\right) d u\right)
$$

The self-similar solutions of the LSW are in the set of variables (2.16) given by the steady states of (2.19). These solutions are given by

$$
\frac{G_{s}(z)}{G_{s}(0)}=\exp \left(-\int_{0}^{z} \frac{d \xi}{1+\beta_{*} H(\xi)}\right)
$$

In particular, the self-similar solution with maximal support is the one for which the velocity of the characteristics vanishes at $z=\infty$, whence $\beta_{*}=-\left(\kappa_{*}-2\right)$. It then follows from (4.11) that

$$
-\left(\kappa_{*}-2\right)=\Phi\left(\int_{0}^{1} u^{-2 / 3}\left(\frac{G_{s}(z)}{G_{s}(0)}-e^{-z}\right) d u\right)
$$

whence, using (4.12) and (4.10), the Lemma follows.

The following result shows that all the characteristic curves associated to (2.19) are strictly decreasing for sufficiently large $\tau$.

Lemma 4.6. Suppose that $\kappa_{*}>4$ and that (4.1) is satisfied. Then there exists $\tau_{0}=\tau_{0}\left(\kappa_{*}\right)>0$ and $\varepsilon_{0}=\varepsilon_{0}\left(\kappa_{*}\right)>0$ such that

$$
\inf _{z>0}\{1+\beta(\tau) H(z)\}>\varepsilon_{0}
$$

for $\tau \geq \tau_{0}$.

Proof. Using (2.19), it follows that $Q=\partial_{z}(\log (G(z, \tau)))$ satisfies in the distribution sense

$$
\begin{aligned}
\partial_{\tau} Q-(1+\beta(\tau) H(z)) \partial_{z} Q & =\beta(\tau) H^{\prime}(z) Q, \tau>0, \quad z>0 \\
Q(z, 0) & =Q_{0}(z)
\end{aligned}
$$

The solutions of (4.15), (4.16) satisfy along characteristics

$$
\begin{aligned}
\frac{\partial \tilde{z}}{\partial \tau} & =-(1+\beta(\tau) H(\tilde{z})) \\
\frac{d Q}{d \tau}(\tilde{z}, \tau) & =\beta(\tau) H^{\prime}(\tilde{z}, \tau) Q(\tilde{z}, \tau)
\end{aligned}
$$


Eliminating $\beta(\tau)$ in these equations we obtain

$$
\frac{1}{Q} \frac{d Q}{d \tau}=-\frac{H^{\prime}(\tilde{z})}{H(\tilde{z})}\left(1+\frac{\partial \tilde{z}}{\partial \tau}\right)
$$

and integrating (4.19) along characteristics gives

$$
\frac{Q(z, \tau)}{Q_{0}\left(\tilde{z}_{0}(z, \tau)\right)}=\frac{H\left(\tilde{z}_{0}(z, \tau)\right)}{H(z)} \exp \left(-\int_{0}^{\tau} \frac{H^{\prime}\left(\tilde{z}\left(s, \tilde{z}_{0}\right)\right)}{H\left(\tilde{z}\left(s, \tilde{z}_{0}\right)\right)} d s\right)
$$

where $\tilde{z}\left(\tau, \tilde{z}_{0}(z, \tau)\right)=z$.

Recalling that $Q=\partial_{z} \log G(z, \tau)$ and integrating (4.20) over $z$ yields

$$
\begin{aligned}
& \frac{G(z, \tau)}{G(0, \tau)}= \\
& \exp \left(\int_{0}^{z} \frac{H\left(\tilde{z}_{0}(\xi, \tau)\right) Q_{0}\left(\tilde{z}_{0}(\xi, \tau)\right)}{H(\xi)} \exp \left(-\int_{0}^{\tau} \frac{H^{\prime}\left(\tilde{z}\left(s, \tilde{z}_{0}(\xi, \tau)\right)\right)}{H\left(\tilde{z}\left(s, \tilde{z}_{0}(\xi, \tau)\right)\right)} d s\right) d \xi\right)
\end{aligned}
$$

Due to Lemma 4.2 we have $\lim _{\tau \rightarrow \infty} \tilde{z}_{0}(\xi, \tau)=\infty$. Therefore, since $Q_{0}<0$ as well as $\exp \left(-\int_{0}^{\tau} \frac{H^{\prime}\left(\tilde{z}\left(s, \tilde{z}_{0}(\xi, \tau)\right)\right)}{H\left(\tilde{z}\left(s, \tilde{z}_{0}(\xi, \tau)\right)\right)} d s\right)<1$ we obtain, using Lemma $4.3,(2.24)$, as well as the fact that $H$ is increasing, that

$$
\lim \inf _{\tau \rightarrow \infty}\left(\frac{G(z, \tau)}{G(0, \tau)}\right) \geq \exp \left(-\int_{0}^{z} \frac{H(\infty)}{H(\xi)} d \xi\right)
$$

Plugging (4.22) into (4.11) and using $H \geq \frac{1}{\kappa_{*}}$ we obtain

$$
\begin{aligned}
1 & +\lim \inf _{\tau \rightarrow \infty}(\beta(\tau)) H(z) \\
& \geq 1-\frac{1}{\kappa_{*}}\left|\Phi\left(\int_{0}^{1} u^{-2 / 3}\left(\exp \left(-\int_{0}^{z} \frac{H(\infty)}{H(\xi)} d \xi\right)-e^{-z}\right) d u\right)\right|
\end{aligned}
$$

Combining this inequality with (4.8) and (4.10), the desired estimate (4.14) follows.

Our next goal is to derive an estimate for $\varepsilon_{0}$ in (4.14) for large $\kappa_{*}$. To this end we compute the asymptotics of the function on the right hand side of (4.23). Moreover, for further reference we compute the asymptotics of a function more general than the one in (4.23). 
Lemma 4.7. The following asymptotic formula is satisfied:

$$
\Phi\left(\int_{0}^{1} u^{-2 / 3}\left(\exp \left(-\int_{0}^{z}\left(\frac{H(\xi)}{H(\infty)}\right)^{\frac{1}{\gamma}-1} d \xi\right)-e^{-z}\right) d u\right) \sim \frac{39}{180}\left(\frac{1}{\gamma}-1\right)
$$

as $\kappa_{*} \rightarrow \infty$, uniformly on sets of the form $0<\delta \leq \gamma \leq \infty$.

Remark 4.8. Notice that $\gamma$ might take the value $\gamma=\infty$, that corresponds to the function in (4.23).

Proof. Using (2.24) and (2.21) we obtain

$$
\frac{H(\xi)}{H(\infty)}=1-\frac{\left(2-v^{1 / 3}\left(1+v^{1 / 3}\right)\right)}{\kappa_{*}}+O\left(\frac{1}{\left(\kappa_{*}\right)^{2}}\right)
$$

as $\kappa_{*} \rightarrow \infty$, where $\xi=\int_{0}^{v} \frac{d y}{f(y)}$. Therefore we obtain

$$
\left(\frac{H(\xi)}{H(\infty)}\right)^{\frac{1}{\gamma}-1}=1-\left(\frac{1}{\gamma}-1\right) \frac{\left(2-v^{1 / 3}\left(1+v^{1 / 3}\right)\right)}{\kappa_{*}}+O\left(\frac{1}{\left(\kappa_{*}\right)^{2}}\right)
$$

uniformly on compact sets of the form $0<\delta \leq \gamma \leq \infty$ as $\kappa_{*} \rightarrow \infty$. Hence

$$
\begin{aligned}
& \int_{0}^{1} u^{-2 / 3}\left(\exp \left(-\int_{0}^{z}\left(\frac{H(\xi)}{H(\infty)}\right)^{\frac{1}{\gamma}-1} d \xi\right)-e^{-z}\right) d u=\int_{0}^{1} u^{-2 / 3} e^{-z} \\
& \cdot\left(\exp \left(\left(\frac{1}{\gamma}-1\right) \int_{0}^{z}\left[\frac{\left(2-v^{1 / 3}\left(1+v^{1 / 3}\right)\right)}{\kappa_{*}}+O\left(\frac{1}{\left(\kappa_{*}\right)^{2}}\right)\right] d \xi\right)-1\right) d u
\end{aligned}
$$

or equivalently

$$
\begin{aligned}
& \int_{0}^{1} u^{-2 / 3}\left(\exp \left(-\int_{0}^{z}\left(\frac{H(\xi)}{H(\infty)}\right)^{\frac{1}{\gamma}-1} d \xi\right)-e^{-z}\right) d u=\int_{0}^{1} u^{-2 / 3} e^{-z} \\
& \left(\exp \left(\left(\frac{1}{\gamma}-1\right) \int_{0}^{u}\left[\frac{\left(2-v^{1 / 3}\left(1+v^{1 / 3}\right)\right)}{\kappa_{*}}+O\left(\frac{1}{\left(\kappa_{*}\right)^{2}}\right)\right] \frac{d v}{f(v)}\right)-1\right) d u
\end{aligned}
$$

Taylor's expansion combined with Taylor's expansion for $f$ ( see (2.13)) 
yields

$$
\begin{aligned}
& \int_{0}^{1} u^{-2 / 3}\left(\exp \left(-\int_{0}^{z}\left(\frac{H(\xi)}{H(\infty)}\right)^{\frac{1}{\gamma}-1} d \xi\right)-e^{-z}\right) d u \\
& =\frac{\left(\frac{1}{\gamma}-1\right)}{\left(\kappa_{*}\right)^{2}} \int_{0}^{1} u^{-2 / 3} e^{-z}\left(\int_{0}^{u} \frac{\left(2-v^{1 / 3}\left(1+v^{1 / 3}\right)\right)}{\left(1-v^{1 / 3}\right)} d v\right) d u+O\left(\frac{1}{\left(\kappa_{*}\right)^{3}}\right) \\
& =\frac{\left(\frac{1}{\gamma}-1\right)}{\left(\kappa_{*}\right)^{2}} \int_{0}^{1} e^{-z}\left(2 u^{1 / 3}+\frac{3}{4} u^{2 / 3}\right) d u+O\left(\frac{1}{\left(\kappa_{*}\right)^{3}}\right)
\end{aligned}
$$

as $\kappa_{*} \rightarrow \infty$. Using (2.17) we find for fixed $u \in(0,1)$ that $e^{-z} \rightarrow 1$ as $\kappa_{*} \rightarrow \infty$. Hence

$$
\begin{gathered}
\int_{0}^{1} u^{-2 / 3}\left(\exp \left(-\int_{0}^{z}\left(\frac{H(\xi)}{H(\infty)}\right)^{\frac{1}{\gamma}-1} d \xi\right)-e^{-z}\right) d u \\
=\frac{39}{20} \frac{\left(\frac{1}{\gamma}-1\right)}{\left(\kappa_{*}\right)^{2}}+O\left(\frac{1}{\left(\kappa_{*}\right)^{3}}\right)
\end{gathered}
$$

whence, using the definition (4.9) of $\Phi,(4.24)$ follows.

We can now derive a uniform lower bound for $\varepsilon_{0}$ in Lemma 4.6.

Lemma 4.9. The constant $\varepsilon_{0}$ satisfies for sufficiently large $\kappa_{*}$ the estimate

$$
\varepsilon_{0} \geq 1-\frac{39}{360} \frac{1}{\kappa_{*}}
$$

Proof. This Lemma follows by combining the asymptotics (4.24) with (4.23).

The following Lemma plays a crucial role in the proof of Theorem 4.1.

Lemma 4.10. Let us define the function.

$$
F(\gamma):=1+\frac{1}{\kappa_{*}-2} \Phi\left(\int_{0}^{1} u^{-2 / 3}\left(\exp \left(-\int_{0}^{z}\left(\frac{H(\xi)}{H(\infty)}\right)^{\frac{1}{\gamma}-1} d \xi\right)-e^{-z}\right) d u\right)
$$

Let us assume that the assumptions of Theorem 4.1 are satisfied. 
(i) Suppose that for $\gamma \in(0,1]$

$$
\lim \inf _{\tau \rightarrow \infty}\left(\inf _{z>0}\{1+\beta(\tau) H(z)\}\right) \geq \gamma
$$

Then $F(\gamma)>1$ and

$$
\lim \sup _{\tau \rightarrow \infty}\left(\sup _{z>0}\{1+\beta(\tau) H(z)\}\right) \leq F(\gamma)
$$

(ii) Suppose that for $\gamma \geq 1$

$$
0<\lim \sup _{\tau \rightarrow \infty}\left(\sup _{z>0}\{1+\beta(\tau) H(z)\}\right) \leq \gamma
$$

Then $F(\gamma) \leq 1$ and

$$
\lim \inf _{\tau \rightarrow \infty}\left(\inf _{z>0}\{1+\beta(\tau) H(z)\}\right) \geq F(\gamma)
$$

Proof. We first notice that $F(1)=1$ and that $F^{\prime} \leq 0$, hence $F$ is decreasing.

We will estimate $\beta$ using (4.11). To that aim we consider formula (4.21) and suppose that (4.27) is satisfied. In the following we omit for an easier reading the arguments in $\tilde{z}_{0}(\xi, \tau)$. We split the integral $\int_{0}^{\tau} \frac{H^{\prime}\left(\tilde{z}\left(s, \tilde{z}_{0}\right)\right)}{H\left(\tilde{z}\left(s, \tilde{z}_{0}\right)\right)} d s$ in (4.21) in the form

$$
\int_{0}^{\tau} \frac{H^{\prime}\left(\tilde{z}\left(s, \tilde{z}_{0}\right)\right)}{H\left(\tilde{z}\left(s, \tilde{z}_{0}\right)\right)} d s=\int_{0}^{\tau_{0}}[\ldots] d s+\int_{\tau_{0}}^{\tau}[\ldots] d s
$$

where $\tau_{0}$ is chosen in such a way that $\inf _{z>0}\{1+\beta(\tau) H(z)\} \geq \gamma-\delta>0$ for some arbitrarily small $\delta$ and for $\tau \geq \tau_{0}$. Notice, that this implies that $\partial_{s} \tilde{z}<0$ for all $s \geq \tau_{0}$. Then

$$
\begin{aligned}
-\int_{\tau_{0}}^{\tau} \frac{H^{\prime}\left(\tilde{z}\left(s, \tilde{z}_{0}\right)\right)}{H\left(\tilde{z}\left(s, \tilde{z}_{0}\right)\right)} d s \\
\quad=\int_{\tau_{0}}^{\tau} \frac{H^{\prime}\left(\tilde{z}\left(s, \tilde{z}_{0}\right)\right) \partial_{s} \tilde{z}\left(s, \tilde{z}_{0}\right)}{H\left(\tilde{z}\left(s, \tilde{z}_{0}\right)\right)} \frac{d s}{1+\beta(\tau) H\left(\tilde{z}\left(s, \tilde{z}_{0}\right)\right)} \\
\quad \geq \frac{1}{(\gamma-\delta)} \int_{\tau_{0}}^{\tau} \frac{H^{\prime}\left(\tilde{z}\left(s, \tilde{z}_{0}\right)\right) \partial_{s} \tilde{z}\left(s, \tilde{z}_{0}\right)}{H\left(\tilde{z}\left(s ; \tilde{z}_{0}\right)\right)} d s \\
\quad=\ln \left(\left(\frac{H(\xi)}{H\left(\tilde{z}\left(\tau_{0}, \tilde{z}_{0}\right)\right)}\right)^{\frac{1}{\alpha-\delta}}\right)
\end{aligned}
$$


On the other hand the exponential decay of $H^{\prime}$ at infinity implies

$$
\lim _{\tau \rightarrow \infty} \int_{0}^{\tau_{0}} \frac{H^{\prime}\left(\tilde{z}\left(s, \tilde{z}_{0}\right)\right)}{H\left(\tilde{z}\left(s, \tilde{z}_{0}\right)\right)} d s=0
$$

We now use (4.1), (4.32) and Lemma 4.3 in formula (4.21) to find

$$
\lim \sup _{\tau \rightarrow \infty} \frac{G(z, \tau)}{G\left(0^{+}, \tau\right)} \leq \exp \left(-\int_{0}^{z}\left(\frac{H(\xi)}{H(\infty)}\right)^{\frac{1}{\gamma-\delta}-1} d \xi\right)
$$

and since $\delta$ might be chosen arbitrarily small

$$
\lim \sup _{\tau \rightarrow \infty} \frac{G(z, \tau)}{G\left(0^{+}, \tau\right)} \leq \exp \left(-\int_{0}^{z}\left(\frac{H(\xi)}{H(\infty)}\right)^{\frac{1}{\gamma}-1} d \xi\right) .
$$

We use this last formula in (4.11), recall that $H \leq \frac{1}{\kappa_{*}-2}$ and find (4.26). The proof of the second part goes analogously.

\section{End of the Proof of Theorem 4.1.-}

For the iterative sequence $\gamma_{n+1}:=F\left(\gamma_{n}\right)$, with initial value $\gamma_{0}:=\varepsilon_{0}$, where $\varepsilon_{0}$ is as in Lemma 4.6, we need to show that it converges to the fixed point 1 of $F$. If $k s>4$, Lemma 4.6 implies that $\gamma_{0}>0$. For sufficiently large $\kappa_{*}$, Lemma 4.7 implies that $\left|F^{\prime}(\gamma)\right|<1$ for all $\gamma \geq \frac{\gamma_{0}}{2}$. Hence $\lim _{n \rightarrow \infty} \gamma_{n}=1$, which implies $\lim _{\tau \rightarrow \infty} \beta(\tau)=0$. As in the proof of Theorem 3.1 the desired convergence result (4.2) follows.

Remark 4.11. We can prove the global stability result as long as (4.8) and $\left|F^{\prime}\right|<1$ are satisfied. We have carried out numerical simulations which indicate that these conditions are satisfied as long as $\kappa_{*}>3,3$. Since this result is not close to the optimal result $\kappa_{*}>2$ we omit the details of these computations.

\section{Estimates on the coarsening rate}

In this last chapter we will derive coarsening estimates for the solution even if the data are not regularly varying at the end of their support. We will need for the proof the condition (see (5.2) below) that the variation of the data at the end of their support is bounded by a sufficiently small constant. 
In view of (2.17) condition (5.2) means that there needs to exist $u_{*} \in(0,1)$ such that for all $u, \hat{u} \geq u_{*}|u-\hat{u}| \leq \frac{1-u_{*}}{\alpha}$

$$
\frac{\left|F_{0}(u)(1-u)^{-\alpha}-F_{0}(\hat{u})(1-\hat{u})^{-\alpha}\right|}{F_{0}(\hat{u})(1-\hat{u})^{-\alpha}} \leq \nu_{*},
$$

where $\alpha=\frac{3}{\kappa_{*}-2}$. Thus, $F_{0}$ can be oscillating at the end of its support, but the amplitude cannot be too large.

Theorem 5.1. Fix $\kappa_{*}>0$ and suppose that $G(z, \tau)$ is a solution of (2.19) with initial data $G(z, 0)=G_{0}(z)=S_{0}(z) e^{-z}$ and that $S_{0}(z)$ satisfies the following: There exists $z_{*}>0$ such that For any $z, \hat{z} \geq z_{*}$ with $|z-\hat{z}| \leq 1$

$$
\frac{\left|S_{0}(z)-S_{0}(\hat{z})\right|}{S_{0}(\hat{z})} \leq \nu_{*}
$$

for a sufficiently small $\nu_{*}>0$. Furthermore assume that there is $S_{*}>0$ such that

$$
S_{0}(z) \geq S_{*} \quad \text { for } z \in\left(0, z_{*}\right) .
$$

Then there exists $M=M\left(\kappa_{*}, z_{*}, S_{*}, \nu_{*}\right)$ such that

$$
\begin{aligned}
0<\frac{1}{M} & \leq \frac{\partial \tilde{z}}{\partial z_{0}}\left(\tau, z_{0}\right) \leq M \\
|\beta(\tau)| & \leq M
\end{aligned}
$$

Moreover, if we denote as $\tau\left(z_{0}\right)$ the value such that $\tilde{z}\left(\tau\left(z_{0}\right), z_{0}\right)=0$ then

$$
\frac{1}{M} z_{0} \leq \tau\left(z_{0}\right) \leq M z_{0}
$$

Proof. We decompose the proof into several steps.

Step 1: As in Lemma 4.2 we derive the lower bound (4.4), that is $\frac{\partial \tilde{z}}{\partial z_{0}} \geq$ $\frac{\kappa_{*}-2}{\kappa_{*}}$.

Step 2: Next we are going to show that there exists $c_{0}=c_{0}\left(k s, z_{*}\right)>0$ such that

$$
\kappa(\tau) \geq c_{0}
$$

for all $\tau>0$ if $\nu_{*}=\nu_{*}\left(\kappa_{*}, z_{*}\right)$ is sufficiently small.

To prove (5.7) we notice that (4.4) yields $z_{0} \leq \bar{z}_{0}+\frac{\kappa_{*}}{\kappa_{*}-2} z$. This implies, since $G_{0}$ is decreasing, that

$$
\frac{G(z, \tau)}{G\left(0^{+}, \tau\right)}=\frac{G_{0}\left(z_{0}\right)}{G_{0}\left(\bar{z}_{0}\right)} \geq \frac{G_{0}\left(\bar{z}_{0}+\frac{\kappa_{*}}{\kappa_{*}-2} z\right)}{G_{0}\left(\bar{z}_{0}\right)} .
$$


Plugging the last inequality into (2.8) we find

$$
\begin{aligned}
\frac{1}{\kappa(\tau)} & =\frac{3}{\int_{0}^{1} u^{-2 / 3} \frac{G(z, \tau)}{G\left(0^{+}, \tau\right)} d u}-1 \leq \frac{3}{\int_{0}^{1} u^{-2 / 3} \frac{G_{0}\left(\bar{z}_{0}+\frac{\kappa_{*}}{\kappa_{*}-2} z\right)}{G_{0}\left(\bar{z}_{0}\right)} d u}-1 \\
& =\frac{3}{\int_{0}^{1} u^{-2 / 3} e^{-\frac{\kappa_{*}}{\kappa_{*}-2} z \frac{S_{0}\left(\bar{z}_{0}+\frac{\kappa_{*}}{\kappa_{*}-2} z\right)}{S_{0}\left(\bar{z}_{0}\right)} d u}-1 .} .
\end{aligned}
$$

Let $u_{*}$ be the value of $u$ corresponding to $z_{*}$ from assumption (5.2). Then

$$
\begin{aligned}
\int_{0}^{1} & u^{-2 / 3} e^{-\frac{\kappa_{*}}{\kappa_{*}-2} z} \frac{S_{0}\left(\bar{z}_{0}+\frac{\kappa_{*}}{\kappa_{*}-2} z\right)}{S_{0}\left(\bar{z}_{0}\right)} d u \geq \int_{u_{*}}^{1} u^{-2 / 3} e^{-\frac{\kappa_{*}}{\kappa_{*}-2} z} \frac{S_{0}\left(\bar{z}_{0}+\frac{\kappa_{*}}{\kappa_{*}-2} z\right)}{S_{0}\left(\bar{z}_{0}\right)} d u \\
& =\int_{u_{*}}^{1} u^{-2 / 3} e^{-\frac{\kappa_{*}}{\kappa_{*}-2} z} d u+\int_{u_{*}}^{1} u^{-2 / 3} e^{-\frac{\kappa_{*}}{\kappa_{*}-2} z}\left(\frac{S_{0}\left(\bar{z}_{0}+\frac{\kappa_{*}}{\kappa_{*}-2} z\right)}{S_{0}\left(\bar{z}_{0}\right)}-1\right) d u \\
& \geq \delta\left(\kappa_{*}, z_{*}\right)-C \nu_{*},
\end{aligned}
$$

where we used in the last step that

$$
S_{0}\left(z_{*}\right) e^{-\nu_{*}\left(z-z_{*}+1\right)} \leq S_{0}(z) \leq S_{0}\left(z_{*}\right) e^{\nu_{*}\left(z-z_{*}+1\right)}, \quad z \geq z_{*} .
$$

which follows from iterating (5.2). For sufficiently small $\nu_{*}=\nu_{*}\left(\kappa_{*}, z_{*}\right)$ the desired lower bound (5.7) follows. We also notice that $\nu_{*} \rightarrow 0$ as $\kappa_{*} \rightarrow \infty$ or $z_{*} \rightarrow \infty$.

Step 3: The next key step in the proof of the theorem will be to derive an upper bound for

$$
\Phi(z, \tau)=\int_{0}^{\tau} \frac{H^{\prime}\left(\tilde{z}\left(s, z_{0}\right)\right)}{H\left(\tilde{z}\left(s, z_{0}\right)\right)} d s
$$

with $z=\tilde{z}\left(\tau, z_{0}\right)$, which appears in the spreading of the characteristics in (4.6). We first collect some basic properties of $\Phi$. We find

$$
\begin{aligned}
\Phi(z, \tau)>0, & \tau>0, z>0, \\
\lim _{z \rightarrow \infty} \Phi(z, \tau)=0, & \tau>0, \\
\Phi(\cdot, \tau) \text { is decreasing } & \text { for } \tau>0 .
\end{aligned}
$$

We notice that (5.11) is just a consequence of the fact that $H^{\prime}(z)$ decays exponentially as $z \rightarrow \infty$. In order to show (5.12) it is convenient to compute

$$
F(z):=\frac{H^{\prime}(z)}{H(z)} \stackrel{(2.23)}{=}\left(1-u^{1 / 3}\right)\left(\frac{1}{3} u^{-2 / 3}+\frac{2}{3} u^{-1 / 3}\right)=\frac{1}{3}\left(u^{-2 / 3}+u^{-1 / 3}-2\right) .
$$


Hence

$$
F^{\prime}(z)=-\frac{1}{9}\left(2 u^{-5 / 3}+u^{-4 / 3}\right) \frac{d u}{d z}<0,
$$

and (5.12) follows.

It turns out that $\Phi(\cdot, \tau)$ does not decrease too fast. Indeed, differentiating (5.9) it follows that

$$
\frac{\partial \Phi}{\partial z}=\int_{0}^{\tau} F^{\prime}\left(\tilde{z}\left(s, \tilde{z}_{0}(z, \tau)\right)\right) \frac{\partial \tilde{z}}{\partial z_{0}}\left(s, \tilde{z}_{0}(z, \tau)\right) \frac{\partial \tilde{z}_{0}}{\partial z}(z, \tau) d s,
$$

where $\tilde{z}\left(\tau, \tilde{z}_{0}(z, \tau)\right)=z$. Using (4.6) we obtain

$$
\begin{aligned}
& \frac{\partial \tilde{z}}{\partial z_{0}}\left(s, \tilde{z}_{0}(z, \tau)\right) \frac{\partial \tilde{z}_{0}}{\partial z}(z, \tau) \\
& =\frac{H\left(\tilde{z}\left(s, \tilde{z}_{0}(z, \tau)\right)\right)}{H\left(\tilde{z}_{0}(z, \tau)\right)} \exp \left(\int_{0}^{s} F\left(\tilde{z}\left(t, \tilde{z}_{0}(z, \tau)\right)\right) d t\right) \\
& \quad \cdot \frac{H\left(\tilde{z}_{0}(z, \tau)\right)}{H(z)} \exp \left(-\int_{0}^{\tau} F\left(\tilde{z}\left(t, \tilde{z}_{0}(z, \tau)\right)\right) d t\right) \\
& =\frac{H\left(\tilde{z}\left(s, \tilde{z}_{0}(z, \tau)\right)\right)}{H(z)} \exp \left(-\int_{s}^{\tau} F\left(\tilde{z}\left(t, \tilde{z}_{0}(z, \tau)\right)\right) d t\right),
\end{aligned}
$$

whence

$$
\begin{aligned}
& \frac{\partial \Phi}{\partial z}= \\
& \int_{0}^{\tau} F^{\prime}\left(\tilde{z}\left(s, \tilde{z}_{0}(z, \tau)\right)\right) \frac{H\left(\tilde{z}\left(s, \tilde{z}_{0}(z, \tau)\right)\right)}{H(z)} \exp \left(-\int_{s}^{\tau} F\left(\tilde{z}\left(t, \tilde{z}_{0}(z, \tau)\right)\right) d t\right) d s .
\end{aligned}
$$

Due to $F>0$ and $F^{\prime}<0$ (cf. (5.14)) we have

$$
\frac{\partial \Phi}{\partial z} \geq-\int_{0}^{\tau}\left|F^{\prime}\left(\tilde{z}\left(s, z_{0}(z, \tau)\right)\right)\right| \frac{H\left(\tilde{z}\left(s, \tilde{z}_{0}(z, \tau)\right)\right)}{H(z)} d s .
$$

Step 4: As a first intermediate step we show that there exists a time $\tau_{*}=$ $\tau_{*}\left(\kappa_{*}, z_{*}, S_{*}, \nu_{*}\right)$ such that all characteristics with initial value $z_{0} \leq z_{*}$ reach zero at a time $\tau \leq \tau_{*}$.

We first notice that (5.7) and the continuity of $H$ imply that there exists $\bar{z}>0$ such that $\frac{\partial \tilde{z}}{\partial \tau} \leq-\frac{c_{0}}{2 \kappa_{*}}<0$ for all $z \leq \bar{z}$. In particular, any characteristic which reaches $z=\bar{z}$ vanishes within a time interval of length $\frac{2 \kappa_{*} \bar{z}}{c_{0}}=: \bar{C}$.

Now let $\bar{u}$ be the value of $u$ corresponding to $\bar{z}$ via (2.16). From the volume conservation (2.28) and (3.9), i.e. $G(z, \tau)=G_{0}\left(z_{0}\right) e^{\tau}$, we deduce

$$
1=\int_{0}^{1} G(z, \tau) d u \geq \int_{0}^{\bar{u}} G(z, \tau) d u \geq \bar{u} e^{\tau} G\left(\tilde{z}_{0}(\bar{z}, \tau), \tau\right) .
$$


Furthermore assumption (5.3) implies

$$
\bar{u} e^{\tau} G\left(\tilde{z}_{0}(\bar{z}, \tau), \tau\right) \geq \bar{u} e^{\tau} S_{*} e^{-\tilde{z}_{0}(\bar{z}, \tau)} .
$$

Consequently

$$
\tilde{z}_{0}(\bar{z}, \tau) \geq \ln \left(\frac{\bar{u} e^{\tau}}{S_{*}}\right)=\ln \left(\frac{\bar{u}}{S_{*}}\right)+\tau .
$$

Thus, characteristics with $z_{0} \leq z_{*}$ reach the line $z=\bar{z}$ at a time $\tau \leq$ $z_{*}-\ln \left(\frac{\bar{u}}{S_{*}}\right)$ and disappear for $\tau \leq \tau_{*}:=z_{*}-\ln \left(\frac{\bar{u}}{S_{*}}\right)+\bar{C}$.

Step 5: We are now considering times $\tau$ such that $\tau \geq \tau_{*}$ and show that there exists $\bar{\nu}_{*}>0$ and $M_{0}>0$ such that if $\nu_{*} \leq \bar{\nu}_{*}$ and $\Phi(0, \tau) \geq M_{0}$ then it follows that $\beta(\tau)>0$.

From (3.7) it follows for $z=0$, since $H(0)=\frac{1}{\kappa_{*}}$, that

$$
\frac{\partial \tilde{z}}{\partial \tau}=-1-\frac{\beta(\tau)}{\kappa_{*}}=-\frac{\kappa(\tau)}{\kappa_{*}} .
$$

The continuity of $H(z)$ implies that there exists $z_{1} \geq 0$ such that $\partial_{\tau} \tilde{z} \leq$ $-b<0$ for $0 \leq \tilde{z} \leq z_{1}$. Using (2.16) and (2.21) we find that $F^{\prime} \sim-z^{-5 / 3}$ as $z \rightarrow 0^{+}$. Furthermore, due to (5.14), (2.13) and (2.17), the function $F^{\prime}$ decreases exponentially for $z \geq z_{1}$. Combining these estimates with (5.15) and (5.7) we arrive at

$$
\frac{\partial \Phi}{\partial z} \geq-A \Phi-\frac{B}{z^{2 / 3}} \chi_{\left[0, z_{1}\right]}(z)
$$

Integrating (5.16) we obtain that if $\Phi(0, \tau)$ is sufficiently large then

$$
\Phi(z, \tau) \geq \Phi(0, \tau) \frac{e^{-A z}}{2} .
$$

We can now use (5.17) to derive an estimate for $\Phi(0, \tau)$.

Suppose that $\Phi(0, \tau)$ is larger than $M_{0}>0$, which will be made precise later. It follows from (5.17) that $\Phi(z, \tau) \geq c_{Z} M_{0}$ for $0 \leq z \leq Z$ for large $Z$. With (4.6) it follows that

$$
\left|\frac{\partial \tilde{z}_{0}}{\partial z}\right| \leq \delta_{M_{0}}, \text { for } 0 \leq z \leq Z
$$

where $\delta_{M_{0}} \rightarrow 0$ as $M_{0} \rightarrow \infty$. It follows that

$$
\tilde{z}_{0}(\tau, z)-\bar{z}_{0}(\tau) \leq \delta_{M_{0}} z \text { for } \quad 0 \leq z \leq Z .
$$


We can then estimate $\beta(\tau)$ given by

$$
\beta(\tau)=\left(\frac{3}{\int_{0}^{1} u^{-2 / 3} e^{-\left(\tilde{z}_{0}-\bar{z}_{0}\right)} \frac{S_{0}\left(\tilde{z}_{0}\right)}{S_{0}\left(\bar{z}_{0}\right)} d u}-1\right)^{-1}-\left(\frac{3}{\int_{0}^{1} u^{-2 / 3} e^{-z} d u}-1\right)^{-1}
$$

Let us integrate the first integral term:

$$
\begin{aligned}
\int_{0}^{1} u^{-2 / 3} e^{-\left(\tilde{z}_{0}-\bar{z}_{0}\right)} \frac{S_{0}\left(\tilde{z}_{0}\right)}{S_{0}\left(\bar{z}_{0}\right)} d u= & \int_{0}^{1} u^{-2 / 3} e^{-\left(\tilde{z}_{0}-\bar{z}_{0}\right)} d u \\
& +\int_{0}^{1} u^{-2 / 3} e^{-\left(\tilde{z}_{0}-\bar{z}_{0}\right)}\left[\frac{S_{0}\left(\tilde{z}_{0}\right)}{S_{0}\left(\bar{z}_{0}\right)}-1\right] d u .
\end{aligned}
$$

We can estimate the last term in this formula by $C \nu_{*}$. This follows from Step 4 which implies that $\tilde{z}_{0}, \bar{z}_{0} \geq z_{*}$ and from (5.8).

On the other hand

$$
\begin{aligned}
\int_{0}^{1} u^{-2 / 3} e^{-\left(\tilde{z}_{0}-\bar{z}_{0}\right)} d u= & \int_{0}^{1} u^{-2 / 3} d u+\int_{0}^{1} u^{-2 / 3}\left[e^{-\left(\tilde{z}_{0}-\bar{z}_{0}\right)}-1\right] d u \\
=3 & +\int_{\{0 \leq z \leq Z\}} u^{-2 / 3}\left[e^{-\left(\tilde{z}_{0}-\bar{z}_{0}\right)}-1\right] d u \\
& +\int_{\{z>Z\}} u^{-2 / 3}\left[e^{-\left(\tilde{z}_{0}-\bar{z}_{0}\right)}-1\right] d u
\end{aligned}
$$

and using (5.19) it follows that $\left|\int_{\{0 \leq z \leq Z\}} u^{-2 / 3}\left[e^{-\left(\tilde{z}_{0}-\bar{z}_{0}\right)}-1\right] d u\right| \leq C_{Z} \delta_{M_{0}}$. Finally, the integral $\int_{\{z>Z\}} u^{-2 / 3}\left[e^{-\left(\tilde{z}_{0}-\bar{z}_{0}\right)}-1\right] d u$ is small if $Z$ is sufficiently large. Then

$$
\left|\int_{0}^{1} u^{-2 / 3} e^{-\left(\tilde{z}_{0}-\bar{z}_{0}\right)} \frac{S_{0}\left(\tilde{z}_{0}\right)}{S_{0}\left(\bar{z}_{0}\right)} d u-3\right| \leq \varepsilon_{Z}+C_{Z} \delta_{M_{0}}+C \nu_{*}
$$

where $\varepsilon_{Z} \rightarrow 0$ if $Z \rightarrow \infty$. Therefore $\int_{0}^{1} u^{-2 / 3} e^{-\left(\tilde{z}_{0}-\bar{z}_{0}\right)} \frac{S_{0}\left(\tilde{z}_{0}\right)}{S_{0}\left(\bar{z}_{0}\right)} d u$ is arbitrarily close to 3 if $\nu_{*}$ is sufficiently small and $M_{0}$ is sufficiently large. In particular, it follows that under these assumptions $\beta(\tau)>0$ (cf. (5.20)).

Step 6: There exists $M>0$ such that $\Phi(0, \tau) \leq M$ for all $\tau>0$.

Let us suppose that $\Phi(0, \tau)<M_{0}$ for $0 \leq \tau \leq \bar{\tau}$, and that $\Phi(0, \bar{\tau})=M_{0}$. From Step 4 we know that by taking $M_{0}$ sufficiently large we can assume that $\bar{\tau} \geq \tau_{*}$.

Suppose that $\Phi(0, \tau)$ becomes larger than $M_{0}$ for some $\tau>\bar{\tau}$. The definition of $\Phi(z, \tau)$ implies that along characteristics (i.e. for $z_{0}$ fixed):

$$
\Phi\left(\tilde{z}\left(\tau, z_{0}\right), \tau\right)=\int_{0}^{\tau} \frac{H^{\prime}\left(\tilde{z}\left(s, z_{0}\right)\right)}{H\left(\tilde{z}\left(s, z_{0}\right)\right)} d s
$$


where $\tilde{z}\left(s, z_{0}\right)=z$. Hence, using (5.21),

$$
\Phi\left(\tilde{z}\left(\tau, z_{0}\right), \tau\right)=\Phi\left(\tilde{z}\left(\bar{\tau}, z_{0}\right), \bar{\tau}\right)+\int_{\bar{\tau}}^{\tau} \frac{H^{\prime}\left(\tilde{z}\left(s, z_{0}\right)\right)}{H\left(\tilde{z}\left(s, z_{0}\right)\right)} d s
$$

Since $\Phi(\cdot, \tau)$ is decreasing it follows that $\Phi(z, \tau) \leq \Phi(0, \tau)$. As long as $\Phi(0, \tau)$ remains larger than $M_{0}$ we have $\beta(\tau)>0$, or equivalently

$$
\tilde{z}_{s}\left(s, z_{0}\right)=-\left(1+\beta(s) H\left(\tilde{z}\left(s, z_{0}\right)\right)\right)<-1
$$

i.e.

$$
\left|\tilde{z}_{s}\left(s, z_{0}\right)\right|>1
$$

whence, since $H^{\prime}>0$

$$
\begin{aligned}
\int_{\bar{\tau}}^{\tau} \frac{H^{\prime}\left(\tilde{z}\left(s, z_{0}\right)\right)}{H\left(\tilde{z}\left(s, z_{0}\right)\right)} d s & =\int_{\bar{\tau}}^{\tau} \frac{H^{\prime}\left(\tilde{z}\left(s, z_{0}\right)\right)}{H\left(\tilde{z}\left(s, z_{0}\right)\right)}\left(-\tilde{z}_{s}\left(s, z_{0}\right)\right) \frac{d s}{\left(-\tilde{z}_{s}\left(s, z_{0}\right)\right)} \\
& =\int_{\bar{\tau}}^{\tau} \frac{H^{\prime}\left(\tilde{z}\left(s, z_{0}\right)\right)}{H\left(\tilde{z}\left(s, z_{0}\right)\right)}\left(-\tilde{z}_{s}\left(s, z_{0}\right)\right) \frac{d s}{\left|\tilde{z}_{s}\left(s, z_{0}\right)\right|} \\
& \leq \int_{\bar{\tau}}^{\tau} \frac{H^{\prime}\left(\tilde{z}\left(s, z_{0}\right)\right)}{H\left(\tilde{z}\left(s, z_{0}\right)\right)}\left(-\tilde{z}_{s}\left(s, z_{0}\right)\right) d s \\
& =\log \left(\frac{H\left(\tilde{z}\left(\bar{\tau}, z_{0}\right)\right)}{H\left(z\left(\tau, z_{0}\right)\right)}\right) \\
& \leq \log \left(\frac{H(\infty)}{H(0)}\right)
\end{aligned}
$$

Using (5.22) as well as this inequality we obtain that, as long as $\Phi(0, \tau)$ remains larger than $M_{0}$ we have

$$
\begin{aligned}
\Phi\left(\tilde{z}\left(\tau, z_{0}\right), \tau\right) & =\Phi\left(\tilde{z}\left(\bar{\tau}, z_{0}\right), \bar{\tau}\right)+\log \left(\frac{H(\infty)}{H(0)}\right) \leq \\
\Phi(0, \bar{\tau})+\log \left(\frac{H(\infty)}{H(0)}\right) & =M_{0}+\log \left(\frac{H(\infty)}{H(0)}\right)=: M
\end{aligned}
$$

In particular

$$
\Phi(z, \tau) \leq \Phi(0, \tau) \leq M
$$

for all the times $\tau$ such that $\Phi(0, \tau)>M_{0}$. If $\Phi(0, \tau)$ becomes at some point smaller than $M_{0}$ (5.23) holds, and if for any later time $\bar{\tau}$ we obtain again $\Phi(0, \bar{\tau})=M_{0}$ we can repeat the argument to obtain (5.23). Therefore (5.23) holds for arbitrary times $\tau>0$.

With (5.23) we found, using (4.6), an upper bound for $\frac{\partial \tilde{z}}{\partial z_{0}}$ which finishes the proof of (5.4). 
Step 7: In order to show (5.5) we recall that a lower bound for $\beta(\tau)$ follows from (5.7). Furthermore, Step 4 implies that $\tilde{z}_{0}, \bar{z}_{0} \geq z_{*}$ for $\tau \geq$ $\tau_{*}$. Using (5.8) we find $\int_{0}^{1} u^{-2 / 3} e^{-\left(\tilde{z}_{0}-\bar{z}_{0}\right)} \frac{S_{0}\left(\tilde{z}_{0}\right)}{S_{0}\left(\bar{z}_{0}\right)} d u \leq 3-\delta$ for some $\delta>0$ uniformly in $\tau \geq \tau_{*}$. Hence (5.20) implies the upper estimate for $\beta$.

Step 8: It remains to prove (5.6). The first inequality in (5.6) follows from the boundedness of the speed of the characteristics. The second is a consequence of the fact that the characteristics move at a minimum speed in the region $0 \leq z \leq z_{1}$, combined with (5.4) which implies that characteristics which start in points $z_{0,1}, z_{0,2}$ remain at a distance of order $\left|z_{0,1}-z_{0,2}\right|$ during the whole evolution.

As a consequence of Theorem 5.1 we immediately obtain corresponding coarsening rates for the equation in the original variables. In particular it follows that the largest particle size as well as the mean particle size increase proportional to $t$.

Corollary 5.2. Suppose that $G_{0}(z)$ satisfies the assumptions of Theorem 5.1. Then there exists a constant $M=M\left(\kappa_{*}, z_{*}, S_{*}, \nu_{*}\right)$ such that (recall definition (2.6))

$$
\begin{gathered}
\frac{1}{M} t \leq \bar{v}(t) \leq M t, \quad t>0, \\
\frac{1}{M} t \leq\langle v(t)\rangle:=\frac{\int_{0}^{\infty} F(s, t) d s}{F\left(0^{+}, t\right)} \leq M t, \quad t>0 .
\end{gathered}
$$

Proof. Estimate (5.24) is a consequence of (2.8), (5.5) and (5.7). Using the definitions (2.7) and (3.9) we find

$$
\frac{\int_{0}^{\infty} \varphi(v, t) d v}{\varphi\left(0^{+}, t\right)}=\bar{v}(t) \int_{0}^{1} \frac{G_{0}\left(z_{0}\right)}{G_{0}\left(\bar{z}_{0}\right)} d u=\bar{v}(t) \int_{0}^{1} e^{-\left(z_{0}-\bar{z}_{0}\right)} \frac{S_{0}\left(z_{0}\right)}{S_{0}\left(\bar{z}_{0}\right)} d u .
$$

We can proceed as in the proof of Theorem 5.1 to show that

$$
\lim _{\tau \rightarrow \infty} \int_{0}^{1} e^{-\left(z_{0}-\bar{z}_{0}\right)} \frac{S_{0}\left(z_{0}\right)}{S_{0}\left(\bar{z}_{0}\right)} d u=1 .
$$

Thus, in view of (5.24), estimate (5.25) follows. 


\section{Appendix}

\section{Proof of Lemma 3.3:}

Due to the linearity of the problem it suffices to estimate the corresponding fundamental solution, which is given by

$$
\sigma(\tau, a)=-\int_{0}^{\tau} G(\tau-s) \sigma(s, a) d s+\delta(\tau-a), \quad a>0 .
$$

If we write $\sigma(\tau, a)=\delta(\tau-a)+g(\tau, a)$ then $g$ solves

$$
g(\tau, a)=-\int_{0}^{\tau} G(\tau-s) g(s, a) d s-G(\tau-a) \chi(\tau-a), \quad \tau>0,
$$

where $\chi$ is the characteristic function of $\mathbb{R}^{+}$. Therefore we find $g(\tau, a)=$ $m(\tau-a) \chi(\tau-a)$ where

$$
m(\tau)=-\int_{0}^{\tau} G(\tau-s) m(s) d s-G(\tau), \quad \tau>0 .
$$

We notice that $m\left(0^{+}\right)<0$. Suppose that $m(\tau)<0$ in an interval $\left[0, \tau_{1}\right)$. Then $|m(\tau)|<G(\tau)$ for $\tau \in\left[0, \tau_{1}\right)$. If $\tau_{1}=\infty$ we would obtain $|m(\tau)|<$ $G(\tau)$ for any $\tau>0$ and (3.32) would follow from the representation formula

$$
f(\tau)=\int_{0}^{\infty} \sigma(\tau, a) \lambda(a) d a=\lambda(\tau)+\int_{0}^{\tau} m(\tau-s) \lambda(s) d s .
$$

Let us now assume that $\tau_{1}<\infty$. Denote by $\tau_{1}, \tau_{2}, \ldots, \tau_{n-1}$ the set of values where $m\left(\tau_{k}\right)=0$ and denote $\tau_{0}=0$ and $\tau_{n}=\tau$. Using (6.2) we obtain

$$
\int_{0}^{\tau_{k}} G\left(\tau_{k}-s\right) m(s) d s+G\left(\tau_{k}\right)=-m\left(\tau_{k}\right) \quad k=1,2, \ldots, n,
$$

and

$$
\begin{aligned}
\int_{s \in\left(0, \tau_{k}\right) ; m(s)>0} G\left(\tau_{k}-s\right) m(s) d s & =\int_{s \in\left(0, \tau_{k}\right) ; m(s)<0} G\left(\tau_{k}-s\right)|m(s)| d s \\
& =-G\left(\tau_{k}\right)-m\left(\tau_{k}\right) .
\end{aligned}
$$

Fix $k$ and assume that $m(s)>0$ for $s \in\left(\tau_{k-1}, \tau_{k}\right)$.

Then, since (3.30) implies $\frac{G\left(\tau_{k}-s\right)}{G\left(\tau_{k-1}-s\right)} \leq e^{-\theta\left(\tau_{k}-\tau_{k-1}\right)}$, it follows that

$$
\begin{aligned}
& \int_{s \in\left(0, \tau_{k}\right) ; m(s)>0} G\left(\tau_{k}-s\right) m(s) d s \\
& \leq e^{-\theta\left(\tau_{k}-\tau_{k-1}\right)} \int_{s \in\left(0, \tau_{k-1}\right) ; m(s)<0} G\left(\tau_{k-1}-s\right)|m(s)| d s-G\left(\tau_{k}\right) .
\end{aligned}
$$


If $m(s)<0$ for $x \in\left(\tau_{k-1}, \tau_{k}\right)$ then we obtain

$$
\begin{aligned}
& \int_{s \in\left(0, \tau_{k}\right) ; m(s)<0} G\left(\tau_{k}-s\right)|m(s)| d s \\
& \leq e^{-\theta\left(\tau_{k}-\tau_{k-1}\right)} \int_{s \in\left(0, \tau_{k-1}\right) ; m(s)>0} G\left(\tau_{k-1}-s\right) m(s) d s+G\left(\tau_{k}\right) .
\end{aligned}
$$

Iterating (6.5) and (6.6) we arrive at

$$
\begin{aligned}
& \int_{s \in\left(0, \tau_{n-1}\right) ; m(s) \neq 0} G\left(\tau_{n-1}-s\right)|m(s)| d s \\
& \leq e^{-\theta\left(\tau_{n-1}-\tau_{1}\right)} \int_{0}^{\tau_{1}} G\left(\tau_{1}-s\right)|m(s)| d s \\
& \quad-\quad\left(G\left(\tau_{n-1}\right)-e^{-\theta\left(\tau_{n-1}-\tau_{n-2}\right)} G\left(\tau_{n-2}\right)\right. \\
& \left.\quad+e^{-\theta\left(\tau_{n-1}-\tau_{n-3}\right)} G\left(\tau_{n-3}\right) \cdots e^{-\theta\left(\tau_{n-1}-\tau_{1}\right)} G\left(\tau_{1}\right)\right) .
\end{aligned}
$$

On the other hand (6.4) implies that

$$
\begin{aligned}
\left|m\left(\tau_{n}\right)\right| & =|m(\tau)| \\
& \leq e^{-\theta\left(\tau-\tau_{n-1}\right)} \int_{s \in\left(0, \tau_{n-1}\right) ; m(s) \neq 0} G\left(\tau_{n-1}-s\right)|m(s)| d s+\left|G\left(\tau_{n}\right)\right|,
\end{aligned}
$$

whence

$$
\begin{aligned}
|m(\tau)| & \leq e^{-\theta\left(\tau-\tau_{1}\right)} \int_{0}^{\tau_{1}} G\left(\tau_{1}-s\right)|m(s)| d s \\
& -\left(e^{-\theta\left(\tau-\tau_{n-1}\right)} G\left(\tau_{n-1}\right)-e^{-\theta\left(\tau-\tau_{n-2}\right)} G\left(\tau_{n-2}\right)+e^{-\theta\left(\tau-\tau_{n-3}\right)} G\left(\tau_{n-3}\right)\right. \\
& \left.\ldots e^{-\theta\left(\tau-\tau_{1}\right)} G\left(\tau_{1}\right)\right)+G(\tau) .
\end{aligned}
$$

Since $|m(s)| \leq G(s)$ for $s \in\left(0, \tau_{1}\right)$ we can estimate the first and the last terms on the right hand side by $G(0) e^{-\tau}$. On the other hand the term in 
brackets can be estimated, using (3.30), as

$$
\begin{aligned}
e^{-\theta \tau} \mid & \left(e^{\theta \tau_{n-1}} G\left(\tau_{n-1}\right)\right. \\
& \left.\quad-e^{\theta \tau_{n-2}} G\left(\tau_{n-2}\right)\right)+\left(e^{\theta \tau_{n-3}} G\left(\tau_{n-3}\right) e^{\theta \tau_{n-4}} G\left(\tau_{n-4}\right)\right)+\ldots \mid \\
= & e^{-\theta \tau}\left|\int_{\tau_{n-2}}^{\tau_{n-1}} \frac{d}{d s}\left(e^{\theta s} G(s)\right) d s+\int_{\tau_{n-4}}^{\tau_{n-3}} \frac{d}{d s}\left(e^{\theta s} G(s)\right)+\ldots\right| \\
= & e^{-\theta \tau}\left|\int_{\tau_{n-2}}^{\tau_{n-1}}\right| \frac{d}{d s}\left(e^{\theta s} G(s)\right)\left|d s+\int_{\tau_{n-4}}^{\tau_{n-3}}\right| \frac{d}{d s}\left(e^{\theta s} G(s)\right)|d s+\ldots| \\
\leq & e^{-\theta \tau} \int_{0}^{\tau}\left|\theta e^{\theta s} G(s)+e^{\theta s} G^{\prime}(s)\right| d s \\
\leq & C e^{-\theta \tau} \tau .
\end{aligned}
$$

Summarizing these estimates we finally find

$$
|m(\tau)| \leq C e^{-\frac{\theta}{2} \tau}, \quad \tau>0,
$$

and combining this estimate with the representation formula (6.3) the desired inequality (3.32) follows.

Acknowledgments. The authors gratefully acknowledge support through the DFG-Priority research program SPP 1095 and DGES Grant MTM200405634 .

\section{References}

[1] J. Carr and O. Penrose. Asymptotic behaviour in a simplified Lifshitz-Slyozov equation. Physica D, 124:166-176, 1998.

[2] J. Carrillo and T. Goudon. A numerical study on large-time asymptotics of the Lifshitz-Slyozov system. J. Sc. Comp., 20 1:69-113, 2004.

[3] S. Dai and R. L. Pego. Universal bound on coarsening rates for mean-field models of phase transitions. SIAM J. Math. Anal. to appear.

[4] S. Dai and R. L. Pego. An upper bound on the coarsening rate for mushy zones in a phase-field model. Interfaces and Free Boundaries, 2005. to appear.

[5] B. Giron, B. Meerson, and P. V. Sasorov. Weak selection and stability of localized distributions in Ostwald ripening. Phys. Rev. E, 58:4213-6, 1998.

[6] R. V. Kohn and F. Otto. Upper bounds for coarsening rates. Comm. Math. Phys., 229:375-395, 2002. 
[7] R. V. Kohn and X. Yan. Coarsening rates for models of multicomponent phase separation. Interfaces and Free Boundaries, 6:135-149, 2004.

[8] I. M. Lifshitz and V. V. Slyozov. The kinetics of precipitation from supersaturated solid solutions. J. Phys. Chem. Solids, 19:35-50, 1961.

[9] B. Niethammer and R. L. Pego. Non-self-similar behavior in the LSW theory of Ostwald ripening. J. Stat. Phys., 95, 5/6:867-902, 1999.

[10] B. Niethammer and R. L. Pego. On the initial-value problem in the LifshitzSlyozov-Wagner theory of Ostwald ripening. SIAM J. Math. Anal., 31,3:457$485,2000$.

[11] B. Niethammer and R. L. Pego. The LSW model for domain coarsening: Asymptotic behavior for total conserved mass. J. Stat. Phys., 104, 5/6:11131144, 2001.

[12] B. Niethammer and R. L. Pego. Well-posedness for measure transport in a family of nonlocal domain coarsening models. Indiana Univ. Math. J., 54, 2:499-530, 2005.

[13] B. Niethammer and J. J. L. Velázquez. On the convergence towards a smooth self-similar solution in the LSW model. Preprint.

[14] C. Wagner. Theorie der Alterung von Niederschlägen durch Umlösen. $Z$. Elektrochemie, 65:581-594, 1961. 